\title{
Vibrational Coherence Due to Promoting Mode Activity in the Relaxation Dynamics of the Class III Mixed-Valence Molecule $\left[\mathrm{Ru}_{2} \mathrm{TIEDCl}_{4}\right]^{+}$
}

\author{
Timothy W. Marin, Bradley J. Homoelle, Kenneth G. Spears,* and Joseph T. Hupp* \\ Northwestern University Department of Chemistry, Evanston, Illinois 60208
}

\author{
Larry O. Spreer \\ University of the Pacific, Stockton, California 95211
}

Received: June 27, 2001; In Final Form: November 6, 2001

\begin{abstract}
We present the first measurements of the excited-state relaxation dynamics of a bimetallic class III mixedvalence molecule. The $800 \mathrm{~nm}$ absorption of $\left[\mathrm{Ru}_{2} \mathrm{TIEDCl}_{4}\right]^{+}(\mathrm{TIED}=$ tetraiminoethylenedimacrocycle) relaxes in 250 and 1000 fs to at least two different intermediate states that can be followed with transient absorption spectroscopy. These states decay in 1.3 and $11.5 \mathrm{ps}$, and the absorption of the $1.3 \mathrm{ps}$ intermediate displays a large amplitude, very low frequency, highly damped vibrational coherence that completely modulates the absorption. The coherence frequency is $20 \pm 5 \mathrm{~cm}^{-1}$, and the dephasing times range from 360 to $730 \mathrm{fs}$ over the wavelength range of the absorption band. The occurrence of a low-frequency coherence at room temperature, the nearly $100 \%$ modulation amplitude, and the phase properties as a function of wavelength are consistent with a nonradiative rate modulation rather than the typical impulsive mechanism that creates a coherent FranckCondon modulation of the absorption. A nonradiative rate modulation can occur from a vibronic coupling mechanism that is created by breakdown of the Born-Oppenheimer approximation. This type of electronic state coupling likely occurs via nontotally symmetric vibrations, and this is the first time domain measure of a vibronic coupling frequency for inorganic complexes. The resonance Raman activity of the ground-state absorption is consistent with very small mode displacements for the optically connected ground and excited states, as expected for a class III molecule. Since similar nonradiative rates are measured for both the optically excited-state and intermediate-state decays, they both require similar energy gaps in the range of 5000-7000 $\mathrm{cm}^{-1}$. With these energy gaps, we infer that vibronic coupling matrix elements from 4500 to $11200 \mathrm{~cm}^{-1}$ can explain the observed nonradiative decay time of $250 \mathrm{fs}$. These experiments show that class III molecules, and probably many other inorganic complexes, can have fast nonradiative decay channels from vibronic coupling when electronic states are available at lower energies. Therefore, applications with such molecules require careful molecular design to compete with or reduce rates of return to the ground state.
\end{abstract}

\section{Introduction}

Molecules containing two or more similar redox centers that exist in formally different oxidation states are classified as mixed-valence molecules. Although mixed-valence molecules have been studied and characterized for decades, ${ }^{1,2}$ new varieties continue to be synthesized and examined both experimentally and theoretically. Examples of molecules of this type include the bimetallic tetraiminoethylenedimacrocycles (Figure 1) synthesized by Spreer and co-workers $\left(\mathrm{M}_{2}\right.$ TIEDL 4 : $\mathrm{L}_{\text {is }} \mathrm{Cl}^{-}, \mathrm{CH}_{3}-$ $\mathrm{CN}$, or dimethylformamide; $\mathrm{M}$ is $\mathrm{Ru}$ or $\mathrm{Fe}) .{ }^{3-8}$ The molecules of this series are similar in many respects to the classic CreutzTaube ion $\left(\left(\mathrm{NH}_{3}\right)_{5} \mathrm{Ru}\right.$-pyrazine- $\left.\mathrm{Ru}\left(\mathrm{NH}_{3}\right)_{5}{ }^{5+}\right),{ }^{9}$ sharing many of the chemical and spectroscopic properties expected for a class III molecule. ${ }^{10}$ In this manuscript, we present the first measurements of excited-state relaxation dynamics in class III molecules and identify a relaxation mechanism that could be important for many types of inorganic complexes.

Electrochemical and spectroscopic studies of $\mathrm{M}_{2} \mathrm{TIEDL}_{4}$ demonstrate the following: (1) Three distinct oxidation states are accessible. ${ }^{4}$ (2) The mixed-valent form of each of these molecules (where the oxidation states of the metal centers are nominally II and III) gives rise to a strikingly narrow, intense

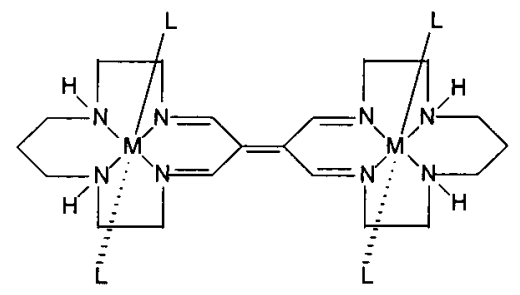

Figure 1. Structure of tetraiminoethylenedimacrocycle, $\left(\mathrm{M}_{2} \mathrm{TIEDL}_{4}\right)^{n+}$, formula $\mathrm{M}_{2} \mathrm{~N}_{8} \mathrm{C}_{20} \mathrm{H}_{36} \mathrm{~L}_{4}$, where $\mathrm{M}=\mathrm{Ru}$ or Fe.

absorption band not found in the fully reduced, isovalent form (oxidation states II and II). ${ }^{6}$ The band occurs in the 800-900 nm range and is assigned as an "intervalence" transition. (3) Analysis of the optical band via Hush methods ${ }^{11}$ implies that the redox state should be described as valence delocalized (Robin and Day class $\mathrm{III}^{10}$ ). The idea of delocalization is strongly supported by several measurements. Electroabsorption measurements ${ }^{12}$ made on $\left[\mathrm{Ru}_{2} \mathrm{TIEDCl}_{4}\right]^{+}$indicate a near-zero change in dipole moment $(\sim 0.1 \mathrm{e} \AA)$ upon formation of the optical excited state at $800 \mathrm{~nm}$. Thus, little charge transfer occurs between the metal centers, implying minimal solvent reorganization energy. Electrochemical experiments ${ }^{5}$ yield an enor- 
mous equilibrium constant $\left(K_{\mathrm{com}}=3.5 \times 10^{15}\right)$ for the mixedvalent comproportionation reaction:

$$
\left[\mathrm{Ru}_{2}^{\mathrm{II}} \mathrm{TIEDCl}_{4}\right]+\left[\mathrm{Ru}_{2}^{\mathrm{III}} \mathrm{TIEDCl}_{4}\right]^{2+} \rightleftharpoons\left[\mathrm{Ru}_{2} \mathrm{TIEDCl}_{4}\right]^{+}
$$

Similar results are found for $\left[\mathrm{Fe}_{2} \mathrm{TIED}\left(\mathrm{CH}_{3} \mathrm{CN}\right)_{4}\right]^{+}{ }^{6}$ Further evidence of the class III status of the complex is provided by $\mathrm{X}$-ray photoelectron measurements. ${ }^{5}$

Though these measurements aid in confirming the class III nature of these molecules, they do not provide insight into the molecular orbitals that give rise to the electronic delocalization properties. Two previously proposed, limiting models offer starting points for describing the strong coupling between metal centers in class III molecules. The first is a two-site vibronic coupling model developed by Piepho, Krausz, and Schatz. ${ }^{13-15}$ The second is a three-site bonding model proposed by Ondrechen and co-workers. ${ }^{16-22}$

Resonance Raman measurements obtained under conditions of optical enhancement clearly indicate that a three-site, rather than two-site, model is needed in order to describe $\left[\mathrm{Fe}_{2}-\right.$ $\left.\mathrm{TIEDCl}_{4}\right]^{+} .{ }^{12}$ This is consistent with earlier resonance Raman studies of the prototypical class III compound, the Creutz-Taube ion. ${ }^{23,24}$ In addition to the prominent optical transition in the near-infrared region, available electronic structure calculations for the Creutz-Taube ion point to the existence of two additional transitions at longer wavelengths. ${ }^{16-22}$ The transitions are associated with electronic promotion from nonbonding d-orbitals localized on a single metal center to an initially half-occupied nonbonding d-orbital shared by the metal centers. The transitions, both of which have been observed experimentally, ${ }^{25}$ are nominally forbidden based on geometric orthogonality of the orbitals but acquire oscillator strength, in part, via spin-orbit coupling interactions. We draw attention to these low-energy transitions and low-lying excited states because such similar states appear to play an important role in the relaxation kinetics upon optical excitation.

Class III molecules such as the $\mathrm{M}_{2}$ TIEDL $_{4}{ }^{n+}$ species have properties that make them interesting candidates for the study of the nonradiative excited-state decay. ${ }^{2,26}$ The most notable properties are the intense low-energy electronic absorption bands that are potentially useful in the context of photochemical energy conversion and exceptionally small ground-state/optical excitedstate vibrational coordinate displacements. The latter translate into exceedingly small absorption Franck-Condon factors (FCF's) and extremely narrow optical absorption line shapes ( $\sim 2300 \mathrm{~cm}^{-1}$ fwhm for $\mathrm{M}=\mathrm{Fe}, \sim 1000 \mathrm{~cm}^{-1}$ for $\mathrm{M}=\mathrm{Ru}$ ). The small FCF's imply very slow rates for direct nonradiative decay from the optical excited state to the electronic ground state. Slow nonradiative relaxation implies intense luminescence (i.e., $k_{\text {radiative }} \gg k_{\text {non-radiative }}$ ), which is not observed experimentally. Therefore, the absence of luminescence implies that one or more intermediate electronic states participate in more rapid optical excited-state relaxation processes.

In the sections that follow, we examine experiments and models for $\left[\mathrm{Ru}_{2} \mathrm{TIEDCl}_{4}\right]^{+}$and discuss what we believe are the first kinetic measurements for optical excited-state relaxation within a class III mixed valence molecule. We find that relaxation occurs on the picosecond time scale and that it is mechanistically complex. Measurements indicate the presence of two observable intermediate states. There is an unusual very low frequency $\left(20 \pm 5 \mathrm{~cm}^{-1}\right)$, high amplitude vibrational coherence in the transient absorption of at least one intermediate state. Evidence suggests that the coherence is due to a modulation of the rate constant between the optically excited state and intermediate state, rather than due to a more typical
TABLE 1: Observed Raman Bands for $\left[\mathrm{Ru}_{2} \mathrm{TIEDCl}_{4}\right]^{+}$and Their Relative Scattering intensities

\begin{tabular}{cc}
\hline $\begin{array}{c}\text { Raman shift } \\
\left(\mathrm{cm}^{-1}\right)\end{array}$ & $\begin{array}{c}\text { relative scattering } \\
\text { intensity }(\mathrm{au})\end{array}$ \\
\hline 534 & 1.00 \\
635 & 0.09 \\
873 & 0.44 \\
975 & 0.04 \\
1047 & 0.16 \\
1558 & 0.21
\end{tabular}

impulsively induced coherence mechanism. In addition, the state with a coherence feature also clearly displays vibrational hot bands, possibly indicating larger mode displacements than the ground-state transition. We discuss how the presence of the coherence features may indicate non-Born-Oppenheimer coupling of the intermediate states to the optically excited state via low-frequency promoting modes. We emphasize the uniqueness of such a coherence effect. Time-dependent analyses of resonance Raman data and FCF calculations are presented to provide a quantitative estimate of the mode displacement values and FCF's for the optical absorption.

\section{Experiental Section}

$\left[\mathrm{Ru}_{2} \mathrm{TIEDCl}_{4}\right]\left(\mathrm{PF}_{6}\right)$ was synthesized according to methods described previously. ${ }^{5,7}$ Preliminary resonance Raman studies showed that $\left[\mathrm{Ru}_{2} \mathrm{TIEDCl}_{4}\right]^{+}$decomposes under irradiation in air-saturated solutions but not in deoxygenated solutions. Thus, air-free samples for Raman measurements were prepared by saturating deoxygenated dry acetonitrile (Fisher, distilled over $\left.\mathrm{CaH}_{2}\right)$ with the solute. The solution was then passed through a Teflon filter with a $0.22 \mu \mathrm{m}$ pore size (Aldrich).

The Raman experiments and interpretation are discussed in Appendix A, and the femtosecond kinetics experiments are discussed in Appendix B.

\section{Results and Discussion}

A. Resonance Raman. The knowledge of the mode displacements and FCF's for the optical absorption facilitates the discussion of the transient absorption measurements and relaxation kinetics by nonradiative transitions. We summarize results from the analysis of resonance Raman data that provide estimates of the mode displacements obtained from Raman and absorption spectral simulations. Appendix A contains details of the analysis.

The relative scattering intensities are listed in Table 1, where values are corrected for self-absorption and instrument response. Raman shifts beyond $2000 \mathrm{~cm}^{-1}$ are unobservable due to the falloff in the Si CCD detector sensitivity in the near-IR region. Similar to the data previously reported for $\left[\mathrm{Fe}_{2} \mathrm{TIEDCl}_{4}\right]^{+}, 12$ six resonantly enhanced modes were observed, indicating that these modes are coupled to the optical transition. Particularly noteworthy is the enhancement of the $534 \mathrm{~cm}^{-1}$ mode previously assigned as a totally symmetric mode of the bis(cyclam) ligand. In analogy to prior work, ${ }^{12}$ this provides compelling qualitative evidence for the validity of a three-center molecular orbital description of the delocalized optical transition.

The data analysis and fitting of the absorption spectrum is explained in Appendix A. Values of the dimensionless displacement $\Delta$ for each mode are shown in Table 2 with their corresponding vibrational coupling constants, $S$, and vibrational reorganization energies, $\lambda_{\text {vib. }}$. Summing the individual $\lambda_{\text {vib }}$ values gives a total vibrational reorganization energy of just $150 \mathrm{~cm}^{-1}$, which is a very small value with errors of about $25 \%$.

B. Transient Absorbance-Kinetic Measurements. B.1. Overview. In this section, we present a brief overview of the 


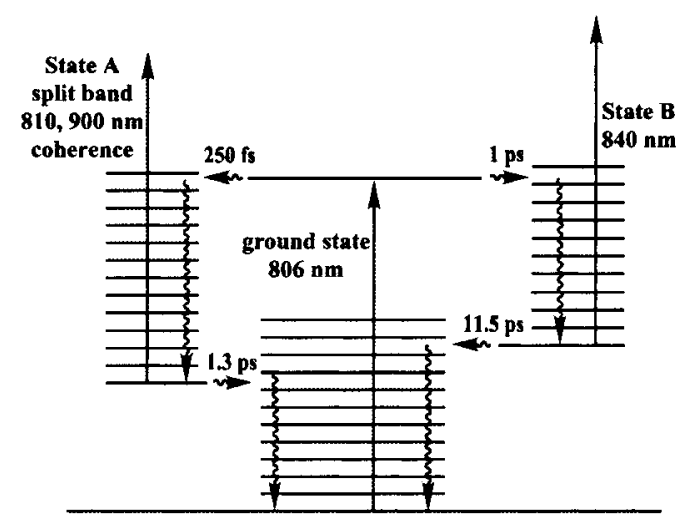

Figure 2. Jablonski diagram summarizing the relaxation dynamics of $\left[\mathrm{Ru}_{2} \mathrm{TIEDCl}_{4}\right]^{+}$.

TABLE 2: Calculated Relative Displacements $(\Delta)$, Vibrational Coupling Constants $(S)$, and Vibrational Reorganization Energies $\left(\lambda_{\text {vib }}\right)$ for the Observed Raman Modes $\left(50\right.$ and $3100 \mathrm{~cm}^{-1}$ Modes Not Experimentally Observed)

\begin{tabular}{cclc}
\hline$v\left(\mathrm{~cm}^{-1}\right)$ & $D$ & \multicolumn{1}{c}{$S$} & $\lambda_{\text {vib }}\left(\mathrm{cm}^{-1}\right)$ \\
\hline 50 & 1.00 & 0.50 & 25 \\
534 & 0.32 & 0.051 & 27 \\
635 & 0.09 & 0.0041 & 3 \\
873 & 0.19 & 0.018 & 16 \\
975 & 0.06 & 0.0014 & 2 \\
1047 & 0.11 & 0.0061 & 6 \\
1558 & 0.13 & 0.0072 & 12 \\
3100 & 0.20 & 0.020 & 62
\end{tabular}

results obtained from transient absorbance experiments. Throughout this section, it will be necessary to refer to a Jablonski diagram in Figure 2 that reflects our conclusions regarding the relaxation dynamics for this molecule. Optical excitation was performed with an $800 \mathrm{~nm}$ pump for all the transient absorbance measurements. The entire wavelength range from 735 to 980 $\mathrm{nm}$ was probed in $\sim 15 \mathrm{~nm}$ increments, thus covering more than the full wavelength range of the ground-state absorption band (the ground-state absorption fwhm is $772-835 \mathrm{~nm}$ with a peak at $806 \mathrm{~nm})$.

The transient absorption in the 735-980 $\mathrm{nm}$ range was complex, and we independently obtained the ground-state recovery kinetics by measuring the bleach recovery kinetics in the MLCT absorption band at 500, 540, and $580 \mathrm{~nm}$. Transient absorption responses were not observed over this wavelength range, indicating that the ground-state bleach was being monitored exclusively. This transient fits a biexponential bleach recovery with time constants of 1.7 and $12.0 \mathrm{ps}$, thereby proving that two channels exist for ground-state recovery following 800 $\mathrm{nm}$ excitation. The ratio of the amplitudes of these components is 4.0 , indicating that approximately $80 \%$ of the excited population relaxes through the 1.7 ps channel. These kinetic values and their relative amplitudes were then held fixed while fitting the more complex transients from 735 to $980 \mathrm{~nm}$.

The complex transients were analyzed and fit with the bleach transients plus two absorption transients having different decays and fast rise times. Also, a coherence feature was present that strongly modulated the transient absorptions. Since the decays of the two transients were very different, it was possible to use their amplitudes from the fitting to plot separate spectra for two intermediate states that are labeled A and B in Figure 2. A split absorption band is observed for state $\mathrm{A}$, with peaks at 810 and $900 \mathrm{~nm}$. The extinction coefficient estimates of these peaks are similar to the ground state, $\sim 50000 \mathrm{M}^{-1} \mathrm{~cm}^{-1}$. State A

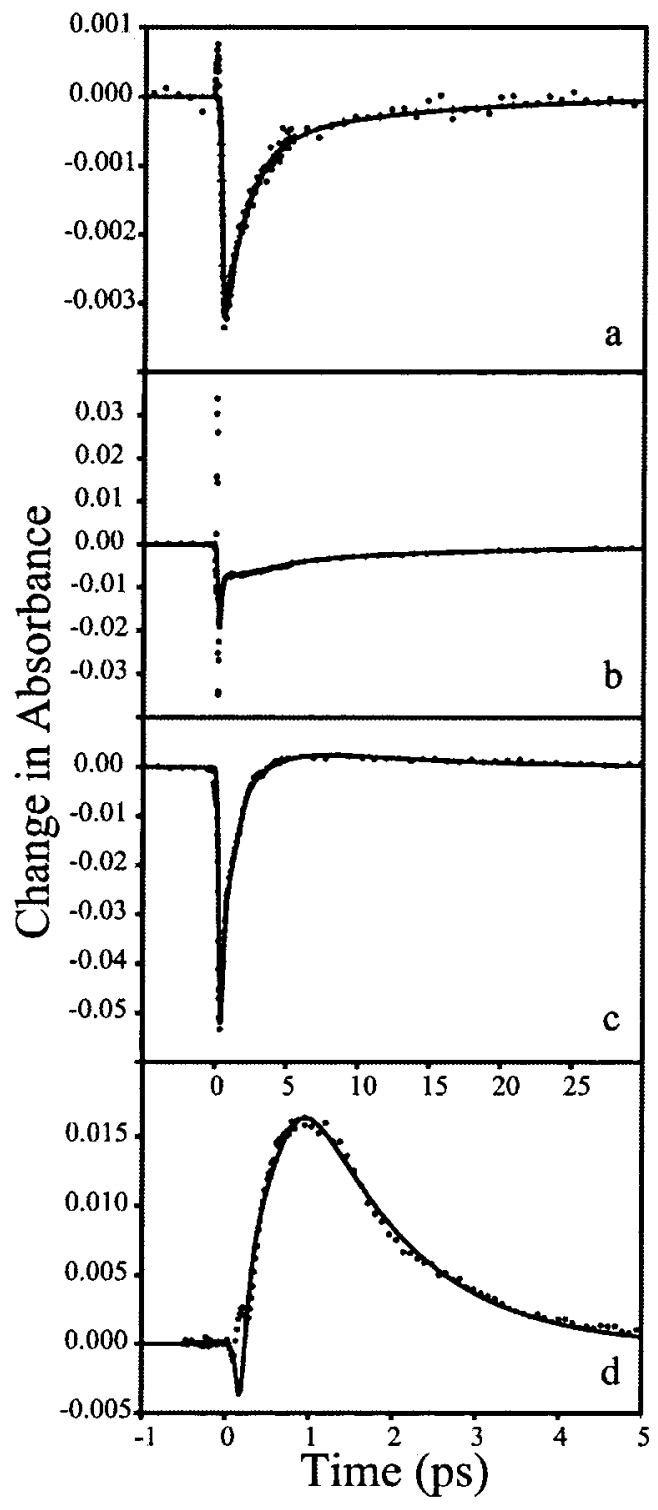

Figure 3. Pump/probe responses of $\left[\mathrm{Ru}_{2} \mathrm{TIEDCl}_{4}\right]^{+} .800 \mathrm{~nm}$ pump and probes at (a) 500, (b) 750, (c) 825 , and (d) $920 \mathrm{~nm}$. Points are raw data, solid lines are fits. A poor fit for panel d is attributed to vibrational relaxation features causing multiexponential decay.

consistently decays with a time constant of $1.3 \mathrm{ps,} \mathrm{while} \mathrm{state}$ $\mathrm{B}$, centered at $840 \mathrm{~nm}$, decays with a time constant of $11.5 \mathrm{ps}$.

An unusual very low-frequency vibrational coherence was observed for state A over the probe wavelength range of 735$920 \mathrm{~nm}$. Fitting gives an average frequency of $20 \pm 5 \mathrm{~cm}^{-1}$ and damping times from 360 to $730 \mathrm{fs}$. The amplitude of the coherence provides $100 \%$ modulation of the transient absorbance at most wavelengths. The modulation is not from stimulated Raman processes, and we find that it arises from modulation of the decay rate constant between the optically excited state and state A. This type of modulation probably originates from electronic coupling by promoting modes in a Born-Oppenheimer breakdown model with nontotally symmetric vibrations.

B.2. Transient Absorptions. Figure 3 displays a selection of transient absorption data at 500 (a), 750 (b), 825 (c), and 920 $\mathrm{nm}$ (d) that each accentuate particular features of the transients. A ground-state bleach recovery (negative absorbance) is observed at all wavelengths, but in the 920 and $825 \mathrm{~nm}$ data, there is clear evidence of transient absorption convoluted with the ground-state dynamics. One can observe a slower decaying transient in the $825 \mathrm{~nm}$ data, while in the expanded time scale 


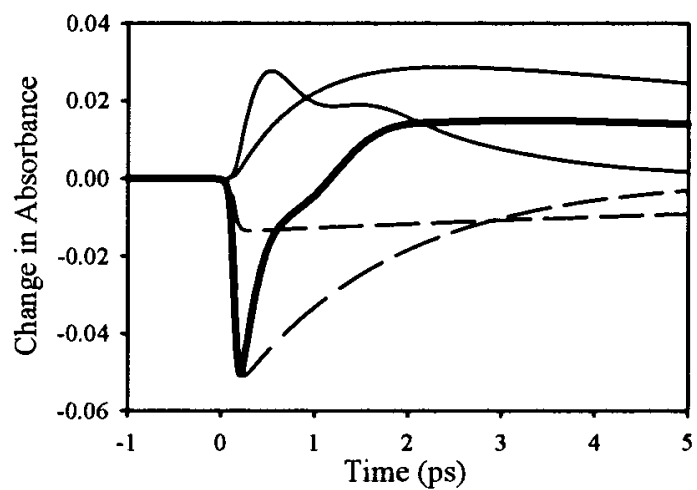

Figure 4. Fit to pump/probe data at $840 \mathrm{~nm}$ showing individual components. Components are 12.0 and 1.7 ps absorption bleaches (dashed lines) and 11.5 and 1.3 ps transient absorptions with rise times (solid lines). Vibrational coherence is included in the $1.3 \mathrm{ps}$ transient. The bold solid line displays the sum of all components.

of panel d, one observes a fast transient at $920 \mathrm{~nm}$. The absorptions are not visually obvious in the $750 \mathrm{~nm}$ data, as the ground-state bleach recovery sums into the total signal in a dominant manner. However, the data precision is sufficient to fit transient features at all wavelengths, and since we have independently obtained the ground-state bleach recovery time constants from the $500 \mathrm{~nm}$ experiments, the transient absorption component can be analyzed with confidence, even in the presence of a strong bleach component.

The data (identical to the bold line) for $840 \mathrm{~nm}$ shown in Figure 4 demonstrates the coherence features of the data with the individual components used for the data fits. This figure illustrates the coherence modulation for component $\mathrm{A}$, and we extensively discuss the fitting of the coherence in a later section. We selected fitting functions to make the most physical sense on the basis of the self-consistency of the model at all probe colors and held parameters fixed whenever possible. The fast and slow transient decays each have a rise time, which suggests that they arise as intermediate states and are not populated by direct excitation. To estimate fitting errors, we have fixed parameters over a range and compared their cross correlations while examining the effect on the reduced chi squared value. Since data were acquired at multiple probe wavelengths over many days, absolute errors in transient absorbance amplitudes might be as large as $25 \%$. The errors in fits to coherence parameters are likely to be $25 \%$, while other kinetic constants are more accurate (see later discussion).

The data shows a fast decaying transient (transient A in Figure 2) for every wavelength, while the slower decaying absorption feature (transient B) is most important throughout the 810$920 \mathrm{~nm}$ range. The transient $\mathrm{B}$ rises in $200 \mathrm{fs}$ and decays in $11.5 \mathrm{ps}$, and its amplitude peaks at $\sim 840 \mathrm{~nm}$ with an estimated extinction coefficient of $\sim 50000 \mathrm{M}^{-1} \mathrm{~cm}^{-1}$. Extinction estimates are from amplitude comparison to the ground-state bleach signals. This transient is the likely source of the 12.0 ps bleach recovery. The transient $\mathrm{A}$ decays in $1.3 \mathrm{ps}$ with an extinction similar to transient $\mathrm{B}$, and it is the likely source of the $1.7 \mathrm{ps}$ bleach recovery.

The relative amplitudes of each transient can be used to plot separate absorption spectra. The ground-state absorption spectrum was used to calibrate the intensity of the bleach features, thereby normalizing other fitting coefficients. The spectrum for transient $\mathrm{A}$ is shown in Figure 5a, transient B in Figure 5b, and the ground-state bleach in Figure $5 \mathrm{c}$ (where the solid line is the actual absorption spectrum). The width of the absorption B is very similar to that of the ground-state band, $\sim 1000 \mathrm{~cm}^{-1} \mathrm{fwhm}$.

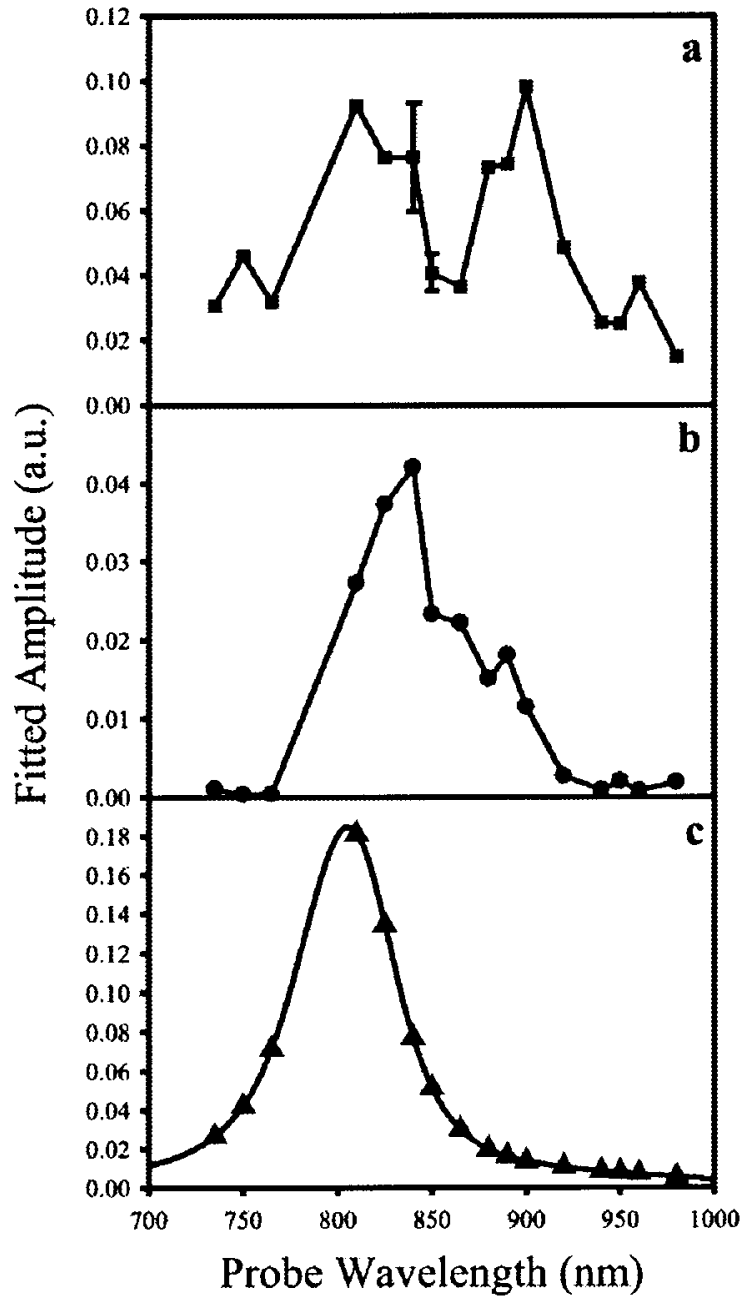

Figure 5. Normalized amplitudes of fitted components versus probe wavelength: (a) state A, (b) state B, and (c) ground-state bleach intensity plotted with experimental ground-state absorption band. Estimated error bars $( \pm 25 \%)$ are displayed for two points in a.

The transient feature A, the transient with coherence features, retains considerable intensity over the entire wavelength range and the band is split, having two peaks with nominal band centers at 810 and $900 \mathrm{~nm}$ and estimated extinction coefficients very similar to state $B$.

At a probe color of $980 \mathrm{~nm}$, the intensity of both the bleach recovery and absorption from state $\mathrm{B}$ are weak enough to attribute nearly all of the transient signal to state A. Here, the rise time is $200 \mathrm{fs}$ and the decay is $950 \mathrm{fs}$. The rise time in the $810-840 \mathrm{~nm}$ region is about $500 \mathrm{fs}$, which includes an average effect from vibrational relaxation (see later discussion). Table 3 lists the rise and decay times for state $\mathrm{A}$ and the rise times for state B where the decay time for state B was held constant at $11.5 \mathrm{ps}$. The vibrational relaxation effects are minimized at the longest wavelength probes, and we therefore use the $200 \mathrm{fs}$ rise time as the intrinsic rate for populating the intermediate state A from some common state reached by the optical pumping at $800 \mathrm{~nm}$ (see later discussion).

The model in Figure 2 shows the competing relaxation from the optically pumped state into the two intermediate states. Since we have an amplitude ratio of $4: 1$ for the fast and slow components of the bleach recovery at $500 \mathrm{~nm}$, this ratio and the $200 \mathrm{fs}$ rise for state A allows estimation of the nonradiative decay rate into state $\mathrm{B}$. The observed rise time of $200 \mathrm{fs}$ for state $\mathrm{A}$ is the sum of these two rates, and therefore the decay times to states A and B are 250 and 1000 fs, respectively. The 
TABLE 3: Parameters from Fits to Pump/Probe Data for the Transient States A and B

\begin{tabular}{cccc}
\hline $\begin{array}{c}\text { probe color } \\
(\mathrm{nm})\end{array}$ & $\begin{array}{c}\text { lifetime }^{a} \text { of } \\
\text { state A (ps) }\end{array}$ & $\begin{array}{c}\text { rise time }^{a} \text { of } \\
\text { state A }(\mathrm{ps})\end{array}$ & $\begin{array}{c}\text { rise time }^{a} \text { of } \\
\text { state B (ps) }\end{array}$ \\
\hline 735 & 1.25 & 0.33 & 0.26 \\
750 & 1.27 & 0.34 & 0.29 \\
765 & 1.33 & 0.36 & 0.28 \\
810 & 1.30 & 0.50 & 0.49 \\
825 & 1.35 & 0.50 & 0.62 \\
840 & 1.32 & 0.50 & 0.79 \\
850 & 1.33 & 0.46 & 0.74 \\
865 & 1.36 & 0.45 & 0.61 \\
880 & 1.30 & 0.37 & 0.46 \\
890 & 1.30 & 0.36 & 0.47 \\
900 & 1.32 & 0.34 & 0.44 \\
920 & 1.30 & 0.30 & 0.27 \\
940 & 1.25 & 0.26 & 0.29 \\
950 & 1.18 & 0.24 & 0.28 \\
960 & 1.12 & 0.20 & 0.20 \\
980 & 0.95 & 0.20 & 0.20
\end{tabular}

${ }^{a}$ Maximum uncertainties in the fitted lifetimes and rise times for states A and B are estimated at $\pm 25 \%$ and $40 \%$, respectively. Rise times less than $200 \mathrm{fs}$ are unobtainable for wavelengths in the 735$765 \mathrm{~nm}$ range due to interference from electronic coherence.

observed rise time for state B should be $200 \mathrm{fs}$ in the absence of any vibrational redistribution (VR) features, but there is an added rise time from VR in most fits to the data.

Since the error bars are large for comparing absolute amplitudes of the fitting functions at different wavelengths, one might question the validity of a split absorption band for state A. However, changing the dual peak into a single, wide peak would require error bars of over $\pm 50 \%$, which is unreasonable. The measurement method used the relative intensity of the ground-state absorption spectrum to calibrate the intensity of the bleach features, thereby normalizing other fitting coefficients. Furthermore, the data collected over the wavelengths where there is a dip in the spectrum $(840-880 \mathrm{~nm})$ were acquired on several different days over a seven-week period using different samples for each wavelength, which supports the validity of two bands. The difference in energy between the peaks at 810 and $900 \mathrm{~nm}$ is $1240 \mathrm{~cm}^{-1}$, which is a moderate value that could range from 1000 to $1300 \mathrm{~cm}^{-1}$, considering the uncertainty in peak locations. The data in Table 3 show similar decay times in the main part of both peaks, but a faster rise time is present in the $900 \mathrm{~nm}$ region. The rise time is less accurate than the decay, with likely errors of $\pm 25 \%$. While the fits show reasonable exponential character in the most intense regions, the signals could contain some multiexponential character from vibrational relaxation kinetics.

The presence of two transient absorption peaks of similar oscillator strength and with very similar relaxation kinetics suggests that the $\sim 1200 \mathrm{~cm}^{-1}$ splitting is in the upper state of the absorption. If the splitting were in the lower state, the decay rate to the ground state of 1.3 ps should be very different with such a large difference in energy gap. The later discussion on vibrational coherence provides further discussion on nonradiative rates and energy gaps.

B.3. Vibrational Relaxation. Both experimental and theoretical issues concerning vibrational relaxation (VR) and intramolecular vibrational redistribution (IVR) have been addressed in recent years, ${ }^{27-40}$ and models have been proposed which allow the temperature of a vibrational hot band to be calculated based on experimental data. ${ }^{41-44}$ Other models exist which demonstrate the vibrational state coupling within the vibrational quasicontinuum that exists in large polyatomic molecules. ${ }^{45,46}$ These models illustrate that as the probe color is tuned toward the zero energy of the absorption band, the relaxation time and rise time for the hot band absorption should become greater. Absorption intensity near the zero energy mostly involves transitions occurring from the lowest vibrational levels, which are the last levels to be populated in a VR process.

In the far red edge $(\geq 920 \mathrm{~nm})$ of the absorption for intermediate state $\mathrm{A}$, both the rise and decay constants are seen to vary with wavelength (see Table 3 ). We attribute this to VR in state A arising from decay out of multiple displaced vibrationally hot modes. The data fits $\geq 920 \mathrm{~nm}$ actually have nonexponential decay, although the kinetics have been reasonably approximated with a single exponential decay and rise. At the farthest red probe color, $980 \mathrm{~nm}$, the transient is not highly convoluted with state B absorption, and it has a decay time constant of $950 \mathrm{fs}$ and rise time of $200 \mathrm{fs}$. Unfortunately, large-amplitude electronic coherence for the wavelengths $735-765 \mathrm{~nm}$ makes it impossible to obtain rise times less than $200 \mathrm{fs}$ in this region.

The data can be interpreted to identify VR times for some states. A ground-state VR time of about $0.5 \mathrm{ps}$ is estimated by comparing the lifetimes of the intermediate states (1.3 and 11.5 $\mathrm{ps})$ with the ground-state recovery times (1.7 and $12.0 \mathrm{ps})$. Direct observation of VR in the ground state is not observable, due in part to the convolution of the bleach signals and transient absorption, but mostly due to a relatively slow transfer from the intermediate states back into the ground state that is spread over many vibrational levels. The rise and decay times for transients A and B should also include a VR time, where we expect maximum values in the band centers. This type of behavior is nominally seen in Table 3 for both transients $\mathrm{A}$ and B. As indicated in prior discussion, state B population is $1 / 4$ of state A, which makes fitting its signal more difficult. Also, there is no wavelength where transient B can be separately fit to check the expected $200 \mathrm{fs}$ rise time based on the kinetic model of Figure 2, which predicts a rise time equivalent to state A. Transient B seems to have rise times near 750 fs rather than the $500 \mathrm{fs}$ of transient A, although we also have greater uncertainties of $40 \%$ for fitting this parameter due to the smaller amplitudes. Likewise, wavelength dependence of the state B decay time is not observable. The longest rise times for state $\mathrm{A}$ show a broad wavelength range with values from 0.36 to 0.50 ps. The rise times do not correlate with the two absorption peaks for state A, suggesting that the splitting is likely due to the upper state. Without knowing the energies of the intermediate states, we only note similarity of the VR times in states A and B and the ground state as 300-500 fs. Later estimates of absolute rates will show that the energy gaps cannot be very different for relaxation to the intermediate states $\mathrm{A}$ and $\mathrm{B}$ and for relaxation from $\mathrm{A}$ and $\mathrm{B}$ to the ground state.

B.4. Vibrational Coherence. Background. When a laser pulse is sufficiently short, it is possible to observe vibrational coherence in a time-resolved measurement. Many groups have examined the presence of coherence features in their data, and this body of work is rapidly increasing now that ultrafast measurements are becoming more common. ${ }^{47-66}$ The reader is directed to theoretical descriptions of vibrational coherence and experiments in the impulsive limit for detailed treatments of these processes. ${ }^{50,52,56,67,60}$ For the case where there is a resonant excitation of a sample, both the ground- and excited-state potential energy surfaces have a role in the creation of coherence. The time evolution of the coherent vibrations can be illustrated with the use of wave packets. A ground-state vibrational coherence is first set up by the vertical excitation of a ground-state wave packet to the excited state. Within the duration of the pump laser pulse, the wave packet propagates 


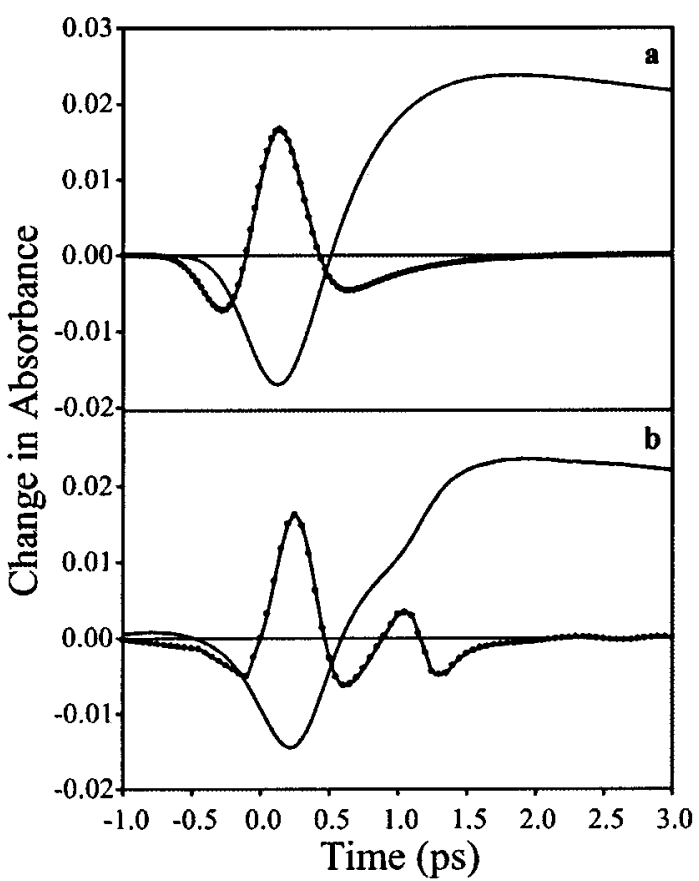

Figure 6. (a) Simulated signal (solid line) and second derivative (solid line with points) for a typical transient signal in the absence of coherence. (b) Smoothed data for $865 \mathrm{~nm}$ probe (solid line) and second derivative (solid line with points). Note the added inflection points in the second derivative of $\mathbf{b}$ where coherence is present

on the excited-state surface and then interacts a second time with the pump field, causing it to be transferred back down to the ground state, now displaced from the absorption maximum. Ground-state coherent effects are then dictated by the oscillation of this wave packet on the ground-state surface, causing periodic fluctuations in the absorption spectrum. This effect is termed impulsively stimulated Raman scattering. For an excited-state coherence effect, the ground-state wave packet is simply excited and begins propagating on the excited-state surface without being stimulated back to the ground state. Oscillatory movement of the wave packet on the excited-state surface then modulates the excited-state spectrum. This process is termed impulsive absorption. Ground-state coherence is observable via absorption bleach measurements, and excited-state coherence is monitored either by excited-state absorption or emission. When on resonance with a transition, the oscillations are expected to be cosinusoidal in nature. Their amplitudes are greatest at the inflection points in the spectrum, occurring on either side of the absorption/emission maximum. At the absorption/emission maximum, the wave packet reaches the energy minimum of the potential energy surface. The coherence amplitude is a minimum at this point and undergoes a $180^{\circ}$ phase shift. ${ }^{53,57,67-69}$ Thus, the phase of the oscillation should be opposite on either side of the absorption maximum with the smallest amplitude coherence at the peak. Larger mode displacements create greater amplitude in the coherence features.

Coherence Observations. Low-frequency, highly damped, oscillatory behavior is observable in intermediate state A that we attribute to vibrational coherence. Figure 6 shows that oscillations are easily observable in the second derivative of the smoothed data. The coherence feature was incorporated into the data fits as a damped cosine function multiplied by a transient absorption decay function with a rise time, $F(t)$

$$
F(t)=A\left(\mathrm{e}^{-k_{1} t}-\mathrm{e}^{-k_{2} t}\right)\left(1+a \cos \left[2 \pi\left(\frac{t}{p}+\frac{\varphi}{360}\right)\right] \mathrm{e}^{-t / d}\right)
$$

TABLE 4: Parameters from Fits to Vibrational Coherence Features $^{a}$

\begin{tabular}{cccccc}
\hline $\begin{array}{c}\text { probe color } \\
(\mathrm{nm})\end{array}$ & $\begin{array}{c}\text { phase shift } \\
(\varphi)(\mathrm{deg})\end{array}$ & $\begin{array}{c}\text { amplitude } \\
(a)\end{array}$ & $\begin{array}{c}\text { period } \\
(\mathrm{ps})\end{array}$ & $\begin{array}{c}\text { frequency } \\
\left(\mathrm{cm}^{-1}\right)\end{array}$ & $\begin{array}{c}\text { dephasing } \\
\text { time }(\mathrm{fs})\end{array}$ \\
\hline 735 & -84 & 0.96 & 1.7 & 20 & 370 \\
750 & -96 & 0.82 & 1.7 & 19 & 400 \\
765 & -132 & 1.00 & 1.6 & 20 & 530 \\
810 & -5 & 1.00 & 2.2 & 15 & 730 \\
825 & -50 & 1.00 & 2.3 & 14 & 710 \\
840 & -48 & 1.00 & 1.7 & 20 & 600 \\
850 & -89 & 1.00 & 2.1 & 16 & 620 \\
865 & -59 & 1.00 & 1.4 & 24 & 420 \\
880 & -115 & 0.87 & 1.4 & 24 & 390 \\
890 & -69 & 0.59 & 1.6 & 21 & 390 \\
900 & 10 & 0.38 & 1.4 & 24 & 360 \\
920 & 49 & 0.59 & 1.6 & 21 & 400
\end{tabular}

${ }^{a}$ Uncertainties in all parameters are $\pm 25 \%$ or less. Due to our fitting function (eq 1), the parameter $a$ is limited to a maximum value of 1 . Where $a$ was converging to near-unity values, it was fixed, thus disallowing a possible convergence on values $>1$.

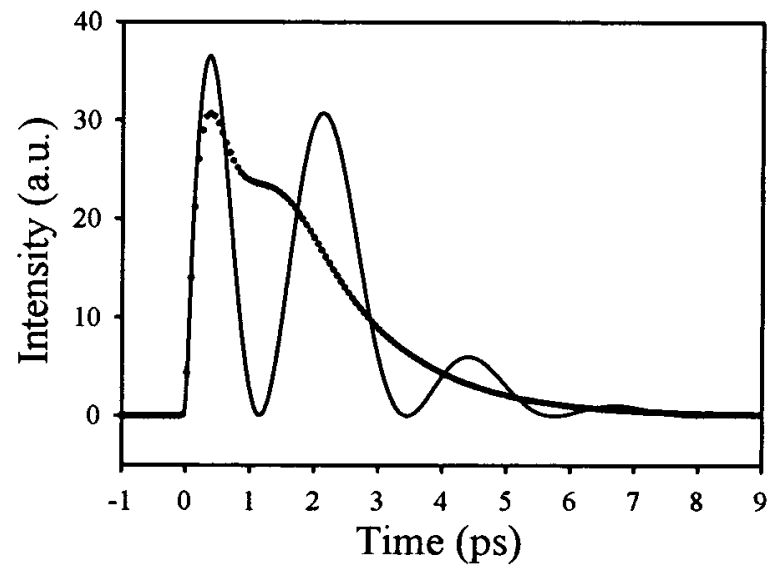

Figure 7. Calculated transient responses showing the effect of dephasing on the apparent amplitude of vibrational coherence. In both responses, the absorption is modulated by $100 \%$. The dotted curve incorporates a $600 \mathrm{fs}$ dephasing time; the solid curve has no dephasing.

Here, $k_{1}$ is the decay constant for the transient, $k_{2}$ is the rise constant, $p$ is the period of the coherence, $\varphi$ is the phase angle in degrees, $d$ is the coherence dephasing time, and $A$ and $a$ ( $a$ can vary between 0 and 1) are amplitude parameters. Previously, Figure 5 was introduced, showing the transient spectral amplitudes versus wavelength, where the displayed amplitude is coefficient $A$ in eq 1. $F(t)$ was fully convoluted with the instrument response function $\left(\mathrm{sech}^{2}\right.$ with typical fwhm of 100 fs). Fitted coherence parameters at each probe color are displayed in Table 4 (estimated errors of $\pm 25 \%$ ). The rise times and decay times of state A are not very wavelength-dependent so that the amplitude of modulation is not dependent on the time parameters. In addition, there is no significant cross correlation between the amplitude parameters $A$ and $a$ in the fitting analysis. Coherence was not incorporated in the data fits at probe wavelengths to the red of $920 \mathrm{~nm}$, as the nonexponential components caused by prominent VR features skew the cosinusoidal behavior. The most unusual feature of the coherence is the $\sim 100 \%$ modulation of the absorption spectrum for state A over the wavelength range of 735-865 nm (i.e., the parameter $a$ fits to a value of 1 and the spectrum modulates between zero intensity and $2 A$ ). The magnitude of the coherence is not visually apparent in the data due to the subpicosecond dephasing times. Figure 7 illustrates a calculated pump/probe signal with parameters similar to those observed for state A (50 fs pump pulse, $0.50 \mathrm{ps}$ rise time, $1.35 \mathrm{ps}$ decay, $2.30 \mathrm{ps}$ coherence 
period). In Figure 7, we show two cases where the coherence modulates the absorbance by $100 \%$. One case has a dephasing time typical of our data (600 fs) and the other has no dephasing. While there is an obvious $100 \%$ absorbance modulation when dephasing is absent, presence of a short dephasing time strongly reduces the apparent amplitude and number of oscillations. The combination of a low frequency and short dephasing time increases the uncertainty in the coherence amplitude. Therefore, to confirm the validity of such a large modulation, we tried many other ways to fit the data. One alternative was to fix $a$ at a smaller value and let the other parameters vary. In this case, even with a large $a=0.5$, the fit lengthens the damping time to increase amplitude in the first peak of the oscillation while having a poor fit at later times $(2-3 \mathrm{ps})$. Another way to check the requirement of a large-amplitude oscillatory function was to use a short transient function to help match the early time data with a smaller amplitude oscillatory function. This also failed to fit well at later times. We also explored simulations involving creation of a low frequency coherence from the beating of two or more high frequency modes. However, the initial large-amplitude in the modulation appears to be a unique constraint, and we always found inadequate fits for any function other than a single coherence function with a large amplitude and fast dephasing time. Assigning the coherence completely to state B does not provide enough amplitude in the oscillations to produce reasonable data fits (see later discussion on partial contributions of B to the larger amplitude of state A). Coherence was not observed in the ground-state bleach recovery. Parameters from the fits are given in Table 4, and they will be discussed in the next section.

Interpretation of Coherence. Several key observations indicate that the coherence does not fit into the impulsive mechanism framework. These are as follows: (1) the coherence amplitude and phase behavior are inconsistent with the impulsive mechanism; (2) the lack of coherence at frequencies greater than 24 $\mathrm{cm}^{-1}$ is unusual when higher frequency modes are active in resonance Raman; (3) the $100 \%$ modulation of the absorption spectrum is unprecedented; (4) the coherence at very low frequencies usually requires low temperatures.

The impulsive mechanism requires that at the absorption/ emission band maximum the coherence amplitude is minimized and that the phase shifts by $180^{\circ}$. On the whole, the phase and amplitude behavior is abnormal when compared with typical impulsive experiments. ${ }^{51-53}$ The clearest deviation from the impulsive mechanism is at the $810 \mathrm{~nm}$ peak where there is large amplitude across the peak. The phase behavior is less clear due to our $25 \%$ fitting errors, although this is more a statistical error rather than an absolute error, which could be larger. There appears to be a phase increase to the red of $810 \mathrm{~nm}$, and by averaging adjacent wavelengths on either side of $810 \mathrm{~nm}(750 /$ 765 and $825 / 840$ ), the phase changes from $-114^{\circ}$ to $-50^{\circ}$. This is not consistent with a $180^{\circ}$ phase shift, but there does seem to be a difference between either side of the peak at $810 \mathrm{~nm}$. The coherence features of the $900 \mathrm{~nm}$ peak are less well defined in our data due to difficulty in fitting the coherence to the red of $920 \mathrm{~nm}$. Therefore, it is not possible to examine an average for several points to the red of $900 \mathrm{~nm}$. In fact, the big change between 880 and 890 suggests that our absolute errors could be larger than $25 \%$ in this region, since we hardly expect such a large effect over $10 \mathrm{~nm}$.

If the coherence were due to the typical Franck-Condon modulation of a displaced totally symmetric mode, it is highly probable that multiple displaced vibrations would be present. Pulses were as short as $50 \mathrm{fs}$ in some experiments, enough to impulsively drive vibrational frequencies as large as $670 \mathrm{~cm}^{-1}$; yet coherence was not observed at frequencies $>24 \mathrm{~cm}^{-1}$. The small mode displacements identified in the resonance Raman analysis of the ground-state absorption suggest that no large coherence should arise from an impulsive event.

The coherence amplitudes of unity are consistent with $100 \%$ modulation of the electronic absorption spectrum. In a model of displacements defining the FCF activity, this implies that a single coherently vibrating mode has the capacity to modulate the entire absorption spectrum. The improbability of having a mode of $20 \mathrm{~cm}^{-1}$ dominate an absorption spectrum arises from several related considerations. The Raman fitting establishes a number of vibrations that are populated in the optically pumped initial excited state, whose subsequent radiationless decay into state A must maintain or increase these populations by FCF's. The absorption spectrum of Figure 5 for state A shows two peaks of similar widths to the ground state, which also suggests that these transient absorptions have vibrational contributions not very different from the ground-state vibrations based on similar modes via the nonradiative process. Therefore, a $20 \mathrm{~cm}^{-1}$ mode that might modulate an absorption spectrum must be a partial contribution to the FCF's contributing to the spectrum, where the incoherent activity provides absorption intensity and greatly reduces the modulation below $100 \%$. Another way to examine the improbability of one mode providing spectral bandwidth is to estimate the required mode displacement. For a $15 \mathrm{~cm}^{-1}$ mode carrying $150 \mathrm{~cm}^{-1}$ of vibrational reorganization energy (value similar to the total vibrational reorganization energy for the ground-state absorption), simple modeling predicts a dimensionless displacement of 4.5 where

$$
\Delta=\sqrt{\frac{2 \lambda_{\mathrm{vib}}}{v}}
$$

Dividing the reorganization energy among three coherent lowfrequency modes still requires large mode displacements on the order of 2.5, and this is too large to be realistic.

The observation of such a low-frequency coherence at room temperature is also unusual, since Boltzmann statistics predict significant population in $\sim 10$ quanta for a $20 \mathrm{~cm}^{-1}$ vibration. Before excitation, these modes in multiple vibrational quanta are all out of phase with each other, and after a vertical excitation, these modes should continue to be out of phase in the optically excited state. Therefore, coherence due to impulsive excitation of such low frequencies should only be observable if the experiments were conducted at very low temperatures, where populations are in only one or two quanta. This hypothesis is supported by previous temperature-dependent experiments, 53 which suggests that the impulsive mechanism is not operative in our experiments.

From these observations, we propose that the coherence does not arise from an impulsive mechanism but rather from modulation of the nonradiative rate constant between the optically excited state and intermediate state A. Recall that a nonradiative rate constant, $k_{\mathrm{nr}}$, in the golden-rule form is written as

$$
k_{\mathrm{nr}}=P_{\mathrm{el}} \times \mathrm{FCWD}
$$

Oscillations in the rate constant can occur either in the electronic prefactor, $P_{\mathrm{el}}$, or in the Franck-Condon weighted density of states, FCWD. Even with large mode displacements, such lowfrequency modes have little effect on the FCWD. Thus, we must consider coherent modulation of the electronic coupling between the states. The modulation is consistent with a low-frequency 


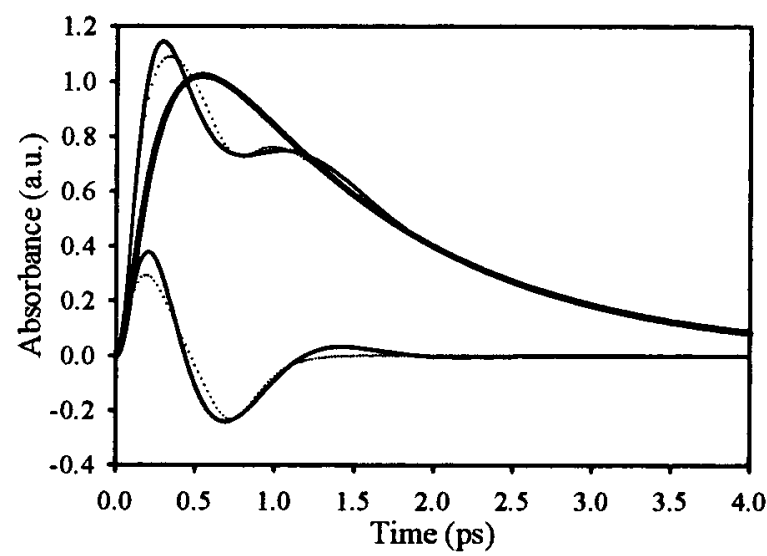

Figure 8. Plot of eq 1 (solid line) and eq 5 (dotted line), showing similarities in the functional form. Values of $k, a, \varphi$, and $p$ are identical for both functions, where $a=1, \varphi=0, p=1.5 \mathrm{ps}, k_{1}=0.77 \mathrm{ps}^{-1}, k_{2}$ $=4.5 \mathrm{ps}^{-1}$, and $d=1.2 \mathrm{ps}$ for eq 5 and $0.5 \mathrm{ps}$ for eq 1 . An unmodulated transient function with identical rate constants is also plotted for comparison (heavy line). The differences between the modulated and unmodulated functions are illustrated as well (lines oscillating around zero value) to show the oscillations.

promoting mode controlling the electronic coupling. This mechanism is a breakdown of the Born-Oppenheimer approximation, often described as a vibronic coupling mechanism. ${ }^{70-74}$ Most likely, promoting modes exist as nontotally symmetric modes in the initial optically pumped state or as acceptors in state A and serve to couple electronic states of different symmetry. The mode activities may arise via torsions in the framework of the molecule that disrupt the $\pi$-bond conjugation or in a complex vibration of the $\mathrm{Ru}-\mathrm{N}$ or $\mathrm{Ru}-\mathrm{Cl}$ bonds. Since there is little solvent reorganization energy for the molecule, a solvent mode is very unlikely. Until more is known about the electronic states, further comments are speculative.

We can demonstrate that a modulated rate constant provides similar observations to the general modulation function of eq 1. A modulated rate constant, $k_{\mathrm{mod}}$, is modeled in our fitting program as

$$
k_{\text {mod }}=k_{2}\left(1+a \cos \left[2 \pi\left(\frac{t}{p}+\frac{\varphi}{360}\right)\right] \mathrm{e}^{-t / d}\right)
$$

where $k$ can either be the rise or decay constant. For our coupling model, $k_{\text {mod }}$ is incorporated into a rise function as

$$
F(t)=A\left(\mathrm{e}^{-k_{1} t}-\mathrm{e}^{-k_{\mathrm{mod}} t}\right)
$$

and similarly can be included into a decay function. As with eq 1 , the amplitude coefficient $a$ can take on any value from 0 to 1 , allowing $k_{\text {mod }}$ to oscillate between values of 0 and $2 k_{2}$. Equation 5 was used to fit transient state $\mathrm{A}$, and results were very similar to those obtained using eq 1 . Within fitting errors, the two functions give similar parameters, except that eq 5 needs longer dephasing times since the rise rate constant $k_{2}$ intrinsically damps out the oscillations. Figure 8 graphically compares eq 1 and eq 5 , indicating that similar oscillatory amplitude and phase can be obtained with both functions for a $22 \mathrm{~cm}^{-1}$ mode. Also, we expect that the effective convolution of population in state A by rise rate constant $k_{2}$ reduces the amplitude of higher frequency oscillations, which would make modulation frequencies higher than $\sim 200 \mathrm{~cm}^{-1}$ unobservable within our signalto-noise levels. Fortuitously, the near equivalence of the modulation frequency and the decay rate creates a large population modulation in the intermediate state.
Equation 5 involves modulation of the rise rate constant to state A rather than a displacement modulation. The rise rate constant modulation arises from vibronic coupling modulation by the coherent low-frequency mode. We know of no mechanism to create a wavelength dependent phase and amplitude from vibronic coupling effects; however, there could be some indirect energy dependence of the coupling mechanism. In addition, perhaps two or more low-frequency modes could be important, and these could even have slightly different frequencies and phases and be associated with the two different peaks. This interpretation has some consistency with our data, but the large errors suggest that the simpler model of a single frequency is most appropriate.

If the rate constant into state $\mathrm{A}$ is modulated, then the complimentary modulated decay rate constant from the optically excited state should also modulate the rise time of state B. However, since the population of transient B is 4 times less than A, we have not attempted to separately add a modulation to the rise time of B due to inadequate signal. Since the state B rise time is 4 times greater than that for state $\mathrm{A}$, the total coherence amplitude fit to state A could actually have $\sim 20 \%$ contribution from state B.

In summary, we have shown that four aspects of the data demonstrate that the coherence is not due to a typical impulsive mechanism. We have suggested that a modulated nonradiative decay rate constant from the optically excited state to intermediate state $\mathrm{A}$ is the likely source of the population modulation of transient A. A rate constant modulation is consistent with a vibronic coupling mechanism where a low-frequency nontotally symmetric promoting mode is responsible for coupling the two states. We emphasize the significance of this effect as, to our knowledge, the rate constant modulation of a nonradiative process has only been observed twice previously. ${ }^{59,75}$ However, the first of these involved a complex with a larger vibrational frequency of totally symmetric character, ${ }^{59}$ and the second a situation where the energy gap was observed to modulate in the absence of solvation effects. ${ }^{75}$ Such high-amplitude, lowfrequency, coherent activity has been previously reported for an intermediate state reached by chemical initiation. ${ }^{61,76}$ Other groups have observed the presence of coherent low-frequency modes either in the ground state or optically excited state, ${ }^{53,77,78,69}$ but most of these arose as small-amplitude features and were assigned to possible impulsive pumping of the sample.

Non-Radiative Rates and Vibronic Coupling. As discussed earlier, a low-frequency coherence of large amplitude is most consistent with a vibronic coupling mechanism involving a promoting mode modulation of the nonradiative rate constant. The intermediate states are probably complex molecular orbitals whose electronic symmetry and spin state are unknown. One expects, similar to models for the Creutz-Taube ion, ${ }^{16-22}$ that these intermediate states could be modeled by vibronic coupling of multiple electronic basis states through high frequency modes of the bridge. While the detailed nature of the intermediate states is unknown, these molecular orbitals can also be vibronically coupled to the optically excited state to provide a mechanism for nonradiative decay. There is a long history of nonradiative decay mechanisms that use vibronic and spin-orbit coupling to explain excited-state conversions as breakdown of the BornOppenheimer approximation. ${ }^{79-86}$ Recently, a model of nonradiative relaxation of ethylene has used ab initio methods ${ }^{71,73}$ to compute vibronic coupling of nontotally symmetric modes in various excited states. If totally symmetric modes are involved as promoting modes, then recent work has shown how displaced modes can be analyzed under certain approximations to provide 
an effective vibronic coupling matrix element that can bridge the domains from strong to weak coupling in a golden-rule expression. ${ }^{87,74}$ We direct the reader to the aforementioned literature for definitions of matrix elements and resulting rate expressions.

For $\left[\mathrm{Ru}_{2} \mathrm{TIEDCl}_{4}\right]^{+}$, we have the likely situation that a lowfrequency motion is breaking electronic symmetry and enabling vibronic coupling of two states. This motion is most likely not a totally symmetric vibration since the intermediate states are not optically visible, and therefore, the intermediate states probably have different symmetry than the optically excited state. The matrix elements for vibronic coupling of nontotally symmetric vibrations can be very large, and values from 1 to 5 $\times 10^{4} \mathrm{~cm}^{-1}$ have been found for ethylene. ${ }^{71,73}$

We can estimate the likely range of coupling matrix elements in $\left[\mathrm{Ru}_{2} \mathrm{TIEDCl}_{4}\right]^{+}$with some assumptions about the mechanism that are consistent with the data. The first point is that the widths of each absorption for state A (see Figure 5a) are similar to those of the ground-state absorption. Therefore, the mode displacements and FCF's are probably similar to those inferred for the optical transition by resonance Raman analysis. This allows an estimate of the FCWD for a given energy gap using the displacements for the optical transition. Given gaps of 5000, 6000 , and $7000 \mathrm{~cm}^{-1}$, we have FCWD values of $1.1 \times 10^{-4}$, $8.6 \times 10^{-5}$, and $1.7 \times 10^{-5}$, respectively. The choice of energy gaps is based on the second observation that the decay from the intermediate states is in the $1-10 \mathrm{ps}$ range, while the decay from the optical state is $250 \mathrm{fs}$. These two times are sufficiently close that each process must have similar energy gaps, so that splitting the $12500 \mathrm{~cm}^{-1}$ gap into $6000 \mathrm{~cm}^{-1}$ for each state is a first approximation. Therefore we assume that an energy gap range of $5000-7000 \mathrm{~cm}^{-1}$ is reasonable for explaining our nonradiative rate of $\sim 5 \times 10^{12} \mathrm{~s}^{-1}$. It should be noted that the calculated value of the $F C W D$ is very sensitive to the estimated displacement $(\Delta=0.20)$ of the added $3100 \mathrm{~cm}^{-1}$ mode. Varying its displacement by \pm 0.05 changes the FCWD by 1 order of magnitude. A high-frequency mode is essential since in its absence the FCWD is reduced by $3-12$ orders of magnitude (the FCWD decreases with increasing energy gap) to the point of requiring unphysical matrix elements to obtain a large nonradiative rate.

To estimate the coupling matrix element consistent with our nonradiate rate, we can use the rate expression developed in eq 3. The electronic prefactor $P_{\mathrm{el}}$ in eq 3 includes an effective coupling matrix element between states a and b as $\left(H_{\mathrm{ab}}\right)_{\mathrm{eff}}$ where

$$
P_{\mathrm{el}}=\frac{2 \pi\left(H_{\mathrm{ab}}\right)_{\mathrm{eff}}^{2}}{\hbar} \frac{1}{\sqrt{4 \pi \lambda_{s} k_{\mathrm{B}} T}}
$$

The term involving temperature and solvent reorganization energy is required by our form of the FCWD. Energy gaps between the optical state and the intermediate state of 5000, 6000 , and $7000 \mathrm{~cm}^{-1}$ require effective coupling matrix elements $\left(H_{\mathrm{ab}}\right)_{\text {eff }}$ with values of 4500,5000 , and $11200 \mathrm{~cm}^{-1}$ to achieve a rate of $5 \times 10^{12} \mathrm{~s}^{-1}$. From analogy with ethylene results ${ }^{71,73}$ that found matrix elements from 1 to $5 \times 10^{4}$, these magnitudes are possible, which suggests that our assumed range of energy gaps of $5000-7000 \mathrm{~cm}^{-1}$ is reasonable.

B.5. Discussion of Mechanism and Experimental Observations. We now summarize the above mechanistic arguments and the coherence features of the transient absorption spectrum. The primary observation is that two transient absorptions exist (Figure 2), where intermediate A has 4 times the population of intermediate $\mathrm{B}$ because of its faster rate for nonradiative decay from the optically pumped state. Intermediate A has a coherence feature of large amplitude and very low frequency of $20 \pm 5$ $\mathrm{cm}^{-1}$. The large amplitude indicates $100 \%$ modulation of the absorption spectrum, which we have shown could be due to vibronic coupling. Plotting the state A amplitude versus wavelength as in Figure 5a reveals two absorption peaks at 810 and $900 \mathrm{~nm}$. The reason for two peaks has not been discussed, and we are not able to distinguish a clear difference in coherence frequency in each peak so have assigned a single coherence frequency to the data.

The first issue is to identify the origin of two absorption peaks for transient absorption A, where each peak in Figure 5a is as narrow as the initial optical absorption. One hypothesis that we must reject is that the peak at $900 \mathrm{~nm}$ is absorption from a vibrationally hot mode with a vibrational frequency of $\sim 1200$ $\mathrm{cm}^{-1}$. If true, then in order to maintain such a narrow transient absorption width similar to the optical absorption from the ground state, this high-frequency mode would have to be a displaced mode significantly different than any found by resonance Raman in the ground state optical absorption. This is unlikely to occur without adding other displaced modes and creating a broader spectrum than the ground-state absorption of Figure 5c. Also, if true, the population of state A would then be partitioned between quantum numbers $v=0$ and $v=1$, and we also would expect $v=2$, which was not observed in our experiments.

Therefore, we must associate the two absorption peaks with two upper states separated by $\sim 1200 \mathrm{~cm}^{-1}$ since the decay of both wavelength regions is the same value of 1.3 ps. State A could be two closely spaced states instead of single state, but any large energy gap difference $\left(>500 \mathrm{~cm}^{-1}\right)$ should give obviously different decay rates back to the ground state. The strong extinction coefficients for the transient absorptions make it unlikely that the coherent promoting mode is involved with controlling the absorption oscillator strength since weakly allowed transitions for conventional molecules usually are made moderately optically allowed, not strongly allowed, by lowfrequency vibronic coupling. ${ }^{8}{ }^{8}$

From the preceding arguments, it is most likely that one state (or two closely spaced states) is associated with intermediate state A. Since the electronic states in this molecule could be complex and their properties highly dependent on symmetry, we cannot be more definite in our conclusions. A provocative conjecture for two moderately spaced states of similar optical absorption strength is a slight asymmetry in the molecular orbitals to create slightly greater electron density around one of the Ru atoms, which would be 2-fold degenerate and undergo vibronic interaction and thereby split into two states. This partial localization could require specific vibrational activity or distortions, or perhaps is an outcome of a complex molecular orbital model. Alternatively, the $\sim 1200 \mathrm{~cm}^{-1}$ splitting could be associated with spin-orbit coupling effects in the two Ru centers which produces two closely lying electronic states. Sophisticated treatments of electronic states will be required to explore these possibilities.

\section{Conclusions}

We have presented the first measurements of electronic relaxation of a class III mixed-valence molecule, $\left[\mathrm{Ru}_{2} \mathrm{TIEDCl}_{4}\right]^{+}$. We find that the optically excited electronic state has two pathways for nonradiative relaxation, each with at least one intermediate electronic state. The initial optically pumped state relaxes into these intermediate states in 250 and $1000 \mathrm{fs}$. Ground-state bleach recovery time constants for the two 
relaxation channels are 1.7 and 12.0 ps. Two intermediate electronic states were observed in the relaxation, the first is at $840 \mathrm{~nm}$ with a decay time constant of $11.5 \mathrm{ps}$; the second is a split band with peaks at 810 and $900 \mathrm{~nm}$ and a decay constant of 1.3 ps.

The transient absorption feature having a 1.3 ps decay has an unusual vibrational coherence feature with a very lowfrequency, highly damped vibration that completely modulates the absorption. The coherence frequency is $20 \pm 5 \mathrm{~cm}^{-1}$, and the dephasing times range from 360 to $730 \mathrm{fs}$ over the absorption band. The occurrence of a low-frequency coherence at room temperature, the $100 \%$ modulation amplitude, and the phase properties as a function of wavelength are consistent with a nonradiative rate modulation rather than the typical impulsive mechanism involving displacement of a totally symmetric mode and Franck-Condon modulation of the absorption spectrum. The nonradiative rate modulation likely occurs via a vibronic coupling mechanism created by a breakdown of the BornOppenheimer approximation. This type of electronic state coupling is probably facilitated with nontotally symmetric vibrations and often has been proposed as an important mechanism for inorganic complexes. This is the first direct measure of a coherent low-frequency mode that facilitates the state coupling.

The observation of two transient absorption bands having identical decay rates suggests that two upper states separated by $\sim 1200 \mathrm{~cm}^{-1}$ provide the band splitting. The complexity of the molecular orbitals for such a large molecule requires advanced theoretical models to identify the experimental features with specific electronic states.

Other experiments and calculations have been carried out to support the kinetic measurements. Resonance Raman data and absorption spectra provide estimates for the small mode displacements and FCF's for the optical absorption. These are consistent with the known class III behavior for this molecule and allow estimates for vibronic coupling matrix elements. Since similar nonradiative rates are measured for both the optically excited-state decay and intermediate state decay, they both require similar energy gaps in the range of $5000-7000 \mathrm{~cm}^{-1}$. With these energy gaps and the FCF estimates we infer that vibronic coupling matrix elements from 4500 to $11200 \mathrm{~cm}^{-1}$ can explain the observed nonradiative decay time of $250 \mathrm{fs}$.

Acknowledgment. We thank Dr. Robert D. Williams for his experimental Raman data on $\left[\mathrm{Ru}_{2} \mathrm{TIEDCl}_{4}\right]^{+}$. We thank Prof. Anne Myers Kelly for the absorption spectrum modeling software. This work was funded by the U.S. Department of Energy, Office of Science (Grants DE-FG02-87ER13808 for J.T.H. and DE-FG02-91ER14228 for K.G.S.).

\section{Appendix A}

Raman Experiments. The Raman excitation source was a Ti:sapphire laser (Spectra Physics Tsunami) running $\mathrm{CW}$ at $820.5 \mathrm{~nm}$ and $300 \mathrm{~mW}$ which was pumped by a multi-line argon ion laser (Coherent Innova 400). The optics were set up in a backscattering configuration. A camera lens was used for light collection and collimation, and an achromatic lens was used for focusing the scattered light through a depolarizer and into the entrance slit of the triple-mate spectrometer (Spex 1877). The spectrometer gratings were blazed at $750 \mathrm{~nm}$. A liquid nitrogen cooled silicon CCD was used for detection. The spectrometer was calibrated for wavelength sensitivity via use of a diffuser and a standardized white light reference lamp (pseudo-blackbody source, Eppley Instruments) featuring a stabilized power supply (Power Ten, Inc.). Correction for selfabsorption was made via the formula ${ }^{89}$

$$
I^{n}=\frac{I_{\mathrm{o}}^{n}}{\left(\frac{\epsilon^{\mathrm{pump}}+\epsilon^{v}}{\epsilon^{\mathrm{pump}}+\epsilon^{v}}\right)}
$$

where $I^{n}$ is the self-absorption corrected intensity of mode $n, I_{\mathrm{o}}^{n}$ is the intensity of mode $n$ without self-absorption correction, $\epsilon$ pump is the extinction coefficient of the probe molecule at the pump wavelength, $\epsilon^{v}$ is the extinction coefficient at the frequency of the mode to which all other mode intensities will be normalized, and $\epsilon_{n}^{v}$ is the extinction coefficient at the frequency of the $n$th mode. The observed Raman bands and relative scattering intensities are given in Table 1.

Resonance Raman Time-Dependent Analysis. The theory and applications of time-dependent Raman scattering have been extensively reviewed..$^{90-98}$ We mention only a few major points to clarify our objectives and approach. It is well-known that the Raman scattering polarizability tensor is primarily related to the mode displacement, $\Delta$, of a vibrational mode. For the transition being resonantly pumped, the relationship between these two is dictated by the half-Fourier transform of the overlap integral involving a wave packet moving on the upper electronic potential energy surface with a vibrational state on the lower surface. The overlap integral can be related to the electronic absorption spectrum via the full Fourier transform.

The analysis here was implemented with a program kindly supplied by the laboratory of Prof. Anne Myers Kelley of Kansas State University. We assumed a temperature of $298 \mathrm{~K}$ and included an additional low-frequency mode (arbitrarily assigned as $\left.50 \mathrm{~cm}^{-1}\right)$ with a relatively large displacement $(\Delta=1.00)$ to allow for energy exchange with solvent modes in the FCF calculations described later. Unfortunately, direct observation of activity in the $3100 \mathrm{~cm}^{-1}$ alkane stretch region was precluded experimentally because of the lack of detector sensitivity at photon energies $<9500 \mathrm{~cm}^{-1}$. To account for possible alkane $\mathrm{C}-\mathrm{H}$ stretching mode activity in the cyclam framework, a highfrequency mode was included at a frequency of $3100 \mathrm{~cm}^{-1}$. Support for the activity in modes of this type was given by an $a b$ initio ground-state vibrational frequencies and Raman intensity calculation on the isovalent molecule with Fe in place of $\mathrm{Ru}$ and without axial ligands. Gaussian $98 \mathrm{~W}$ software ${ }^{99}$ was used with a restricted Hartree-Fock method and basis sets of 3-21G* for $\mathrm{C}$ and $\mathrm{H}, 6-31 \mathrm{G}^{*}$ for $\mathrm{N}$, and 6-311G for Fe; Fe was chosen to replace $\mathrm{Ru}$ as it has fewer electrons, thus expediting such a calculation. Substituting Fe for Ru may significantly affect the calculated Raman intensities; however, the Raman intensities obtained from this calculation were not used in the time-dependent analysis. The purpose was to merely validate the presence of any Raman activity in $\mathrm{C}-\mathrm{H}$ stretching modes. We also find that a high-frequency mode is mandatory in facilitating the relaxation rates, since absence of a $3100 \mathrm{~cm}^{-1}$ mode lowers the calculated rate (see section D6) by 12 orders of magnitude. We note that a single mode is unlikely, so any displacement inferred from the fitting is likely a sum of many modes similar to $3100 \mathrm{~cm}^{-1}$.

The values of $\Delta, E_{00}$ (energy gap), and $\Gamma$ (solvent broadening parameter) were iterated along with homogeneous and inhomogeneous line broadening parameters to produce a reasonable match to our experimental optical absorption spectrum and the relative Raman scattering intensities. For the unknown scattering intensity of the $3100 \mathrm{~cm}^{-1}$ mode, the mode displacement was 


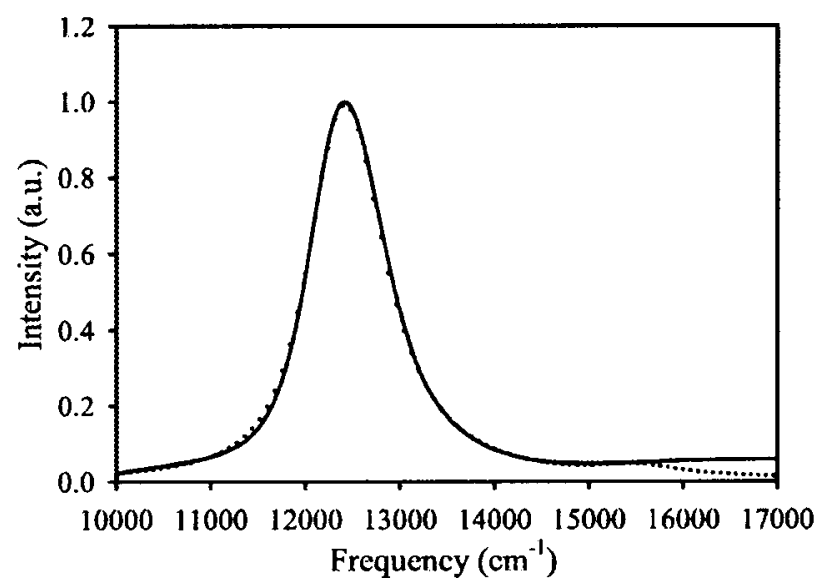

Figure 9. Experimental (solid line) and resonance Raman calculated (dotted line) absorption spectra of $\left[\mathrm{Ru}_{2} \mathrm{TIEDCl}_{4}\right]^{+}$.

iterated to best fit the optical absorption, giving its most noticeable effect on the high-energy edge of the absorption band. Figure 9 shows the fit, where the small peak at high energy suggests that a single mode with this larger displacement should have had an observable peak. As noted above, there is likely more than one mode over a range of frequencies, and this, combined with a peak at $502 \mathrm{~nm}$ contributing to the experimental baseline at $17000 \mathrm{~cm}^{-1}$, does not allow accurate inference of the high-frequency contribution. An outer-sphere solvent reorganization energy, $\lambda_{s}$, is computed in the software from a Brownian oscillator model developed by Mukamel and co-workers. ${ }^{100-102}$ The result of the fit is shown in Figure 9 with parameter values of $\Gamma=450 \mathrm{~cm}^{-1}, E_{00}=12300 \mathrm{~cm}^{-1}$, and $\lambda_{s}=100 \mathrm{~cm}^{-1}$ and inhomogeneous and homogeneous line broadening parameters of 40 and $300 \mathrm{~cm}^{-1}$, respectively. An atypically large homogeneous line broadening of $300 \mathrm{~cm}^{-1}$ is necessary to give a Lorentzian line shape that reproduces the low-energy edge of the absorption spectrum. Values of $\Delta$ for each mode are shown in Table 2 with their corresponding vibrational coupling constants, $S$, and vibrational reorganization energies, $\lambda_{\text {vib. }}$. These values are obtained from the simple formulas $S=1 /{ }_{2} \Delta^{2}$ and $\lambda_{\text {vib }}=S v$, where $v$ is the frequency of the vibration. Summing the individual $\lambda_{\text {vib }}$ values gives a total vibrational reorganization energy of just $150 \mathrm{~cm}^{-1}$. Determination of the absolute mode displacements is precluded by the lack of absolute scattering intensities and we cannot precisely ascertain what fraction of the absorption bandwidth is due to vibronic activity as opposed to broadening effects. The very narrow optical absorption band only constrains the upper limit of the displacements, and an extended vibronic progression is clearly not present for any vibrational mode. We have attempted to increase the displacements while adjusting broadening parameters and find that values greater than $25 \%$ begin to show progressions. Reduction of the displacements was also tested with different broadening parameters. These procedures are only approximate ways to estimate uncertainties, and in this case of a narrow absorption we estimate uncertainties of $\pm 25 \%$ for the mode displacements.

Franck-Condon Analysis for the Optical Absorption. A previously described model for nonadiabatic electron-transfer rates ${ }^{103}$ was used to calculate Franck-Condon factors for the optical transition of $\left[\mathrm{Ru}_{2} \mathrm{TIEDCl}_{4}\right]^{+}$on the basis of the parameters extracted from the Raman analysis/spectral simulation. The individual absorption FCF's for the $534 \mathrm{~cm}^{-1}$ mode (the most highly displaced mode experimentally observed) indicate that $95 \%$ of the spectral intensity lies in $0 \rightarrow 0$ type absorptions, thus supporting the narrow bandwidth of the electronic spectrum. With a candidate assumption of a rate process with an energy gap of $12300 \mathrm{~cm}^{-1}$ and mode displacements equal to those for the optical absorption, we obtain the remarkably small value for the thermally averaged Franck-Condon weighted density of states $(F C W D)$ of $7 \times$ $10^{-9}$, where

$$
\begin{aligned}
& \text { FCWD }= \\
& \qquad \begin{array}{l}
\sum_{v^{\prime}=0}^{\infty}\left|\left\langle v \mid v^{\prime}\right\rangle\right|^{2} \exp \left[-\sum_{j} S_{j} \operatorname{coth}\left(x_{j}\right)\right] \prod_{\substack{j \neq s, m_{m}=-\infty \\
j \neq n}}^{\infty} \exp \left(m_{j} x_{j}\right) I_{\left|m_{m}\right|} z_{j} \\
\exp \left[\frac{-\left(-E_{00}+\lambda_{s}+\sum_{j \neq s} m_{j} \hbar \omega_{j}-v \hbar \omega_{v}+v^{\prime} \hbar \omega_{v^{\prime}}\right)^{2}}{4 \lambda_{s} R T}\right]
\end{array}
\end{aligned}
$$

with

$$
\begin{aligned}
x_{j}=\frac{\hbar \omega_{j}}{2 k_{\mathrm{B}} T} \quad z_{j} & =S_{j} \operatorname{csch}\left(x_{j}\right) \\
I_{m}(z) & =(z / 2)^{m} \sum_{k=0}^{\infty} \frac{\left(z^{2} / 4\right)^{k}}{k !(m+k) !} \quad S_{j}=\frac{f_{j}\left(\Delta Q_{j}\right)^{2}}{2 \hbar \omega_{j}}
\end{aligned}
$$

Here, $v$ is the wave function of the optically excited mode in the ground state with quantum number $v, v^{\prime}$ is the wave function of the optically excited mode in the excited state with quantum number $v^{\prime}, j$ is the number of acceptor modes, $m_{j}$ is the change in acceptor mode quantum number, $s$ is the low-frequency solvent mode, $S_{j}$ is the vibrational coupling constant (HuangRhys factor) for mode $j, I_{m}(z)$ is a modified Bessel function of order $z$, and $f_{j}$ is the force constant for mode $j$ with frequency $\omega_{j} / 2 \pi$ and displacement $\Delta Q_{j}$. The calculated FCWD value is sufficiently small to strongly suggest that for any reasonably large relaxation rate the nonradiative pathway will not be direct to the ground state. The FCWD may have an uncertainty factor of $\sim 5$ based on the uncertainties in the mode displacements reported in section $B$.

\section{Appendix B}

Kinetic measurements were made using conventional pump/ probe methodologies. Our laser system consisted of a modelocked femtosecond Ti:sapphire oscillator ${ }^{104}$ and a pulse stretcher/ regenerative amplifier/pulse compressor ${ }^{105,106}$ for producing high power ultrafast laser pulses. A CW frequency-doubled diodepumped Nd: $\mathrm{YVO}_{4}$ laser (Spectra Physics Millennia) pumped the oscillator with $3.10 \mathrm{~W}$. The oscillator output pulses were centered at $800 \mathrm{~nm}$ with a repetition rate of $83 \mathrm{MHz}$ and 120 $\mathrm{mW}$ of average power $(\sim 1.45 \mathrm{~nJ} /$ pulse). We used a Faraday isolator before a grating pulse stretcher and injected into the regenerative amplifier with a $10 \%$ reflection. The regenerative amplifier was pumped with an average power of $4.15 \mathrm{~W}$ split from a $Q$-switched, frequency-doubled, Nd:YLF laser (Photonics Industries GM-30-527) with $150 \mathrm{~ns}$ pulses at $527 \mathrm{~nm}$ and a 1 $\mathrm{kHz}$ repetition rate. The amplifier cavity-switched output was $750 \mathrm{~mW}$ of $800 \mathrm{~nm}$ pulses at $1 \mathrm{kHz}$. A 4-pass amplifier (Photonics Industries) before the pulse compressor provided additional amplification with little added dispersion. The 4-pass amplifier was pumped with $9.65 \mathrm{~W}$ from the Nd:YLF laser to provide $2.35 \mathrm{~W}$ of $800 \mathrm{~nm}$ pulses at a $1 \mathrm{kHz}$ repetition rate. The amplified beam was split, and each split beam was then sent to a separate grating compressor to independently pump different parametric amplifier systems. Two separate grating 
compressors minimized the thermal instability induced on the grating surface by one high energy beam. Post-compression, pulse widths on the order of $50 \mathrm{fs}$ (fwhm) were observed with a hyperbolic secant squared temporal pulse profile and spectral bandwidth of $27 \mathrm{~nm}$ (fwhm).

Probe pulses were either taken from a white light continuum generated via self-phase modulation of a weak $800 \mathrm{~nm}$ pulse in optical grade sapphire (Crystal Systems) or from the frequency-doubled output of a laboratory-built near-infrared optical parametric amplifier. Pump pulses at $800 \mathrm{~nm}$ were taken directly from the output of a pulse compressor. Pump energies were $4.5 \mu \mathrm{J}$, and probe energies were in the $10 \mathrm{~nJ}$ range. The pump and probe beams were focused at a slight crossing angle of $2^{\circ}$ into the sample to give diameters (fwhm) of 800 and 200 $\mu \mathrm{m}$, respectively. The sample cell was a near-IR transparent quartz cuvette (Aldrich) of $1 \mathrm{~cm}$ path that was sealed with a Teflon coated septum. Post-sample, the probe beam was recollimated and focused into a single-grating imaging spectrometer (Spex 270 M, $1 \mu \mathrm{m}$ blaze grating and modified for thermal stability by removing all electronics from the interior of the spectrometer). A $25 \mathrm{~nm}$ band-pass of the probe beam was selected by the spectrometer and imaged onto the face of a $1 \mathrm{~mm}$ high-speed silicon photodiode (EG\&G FND-100Q, 90 $\mathrm{V}$ bias). In some comparison experiments, the spectrometer was replaced with an interference filter having a $25 \mathrm{~nm}$ band-pass (Corion). Photodiode responses were integrated and processed by means of a preamplifier and amplifier scheme (EG\&G-Ortec $142 \mathrm{~A}$ preamplifier and Ortec 452 spectroscopy amplifier) to produce a linear voltage pulse $(\sim 2 \mu \mathrm{s})$ for A/D conversion. Shotto-shot noise in the probe beam was reduced by measuring a reference beam that had passed through the sample but without overlap with the pump or probe beams. The reference beam was sent through the lower half of the spectrometer entrance slit, imaged onto a separate photodiode, and monitored simultaneously with the probe beam. The ratio of probe to reference intensities was then recorded during data acquisition, giving a much smoother baseline (approximately 10-fold less noise) than given by monitoring the probe intensity alone (relative standard deviation of $\sim 1 \%$ ). Further noise reduction was attained by chopping the pump beam at $0.500 \mathrm{kHz}$ and sequentially subtracting every other shot of the probe beam (pump on, pump off) to perform an actual baseline subtraction that corrects for larger time scale differences between the shots. Normally, 4000 laser shots were averaged per data point, giving a typical baseline noise level of $10^{-4}$ absorbance units.

Three experiments were performed at each wavelength to ensure repeatability of the data. Samples of $\left[\mathrm{Ru}_{2} \mathrm{TIEDCl}_{4}\right]\left(\mathrm{PF}_{6}\right)$ were prepared to have an absorbance of $\sim 1.00$ at $800 \mathrm{~nm}\left(\epsilon_{800}\right.$ $=66300 \mathrm{M}^{-1} \mathrm{~cm}^{-1}$; concentration $\left.\sim 1.5 \times 10^{-5} \mathrm{M}\right)$ in dry, filtered acetonitrile. The solutions were bubbled with purified nitrogen for at least fifteen minutes prior to laser exposure and were kept under nitrogen throughout the experiment to avoid photoinduced degradation. The sample was continuously stirred during the experiment to minimize thermal lensing effects. On the basis of sample concentration and pump laser power densities, we estimate that we normally excited $35 \%$ of the molecules within the sampling volume. Linearity of the transient response was verified at one probe wavelength over the pump energy range of $0.5-9.0 \mu \mathrm{J}$.

Fits to the kinetic data were performed with a laboratory written nonlinear least squares routine and a LevenbergMarquardt fitting algorithm. Convolution of the excitation pulse was incorporated as a $\operatorname{sech}^{2}$ response function. Every kinetic component was fully convoluted with the excitation pulse.
At some wavelengths, oscillatory absorbance features in the data near time zero (the peak of the excitation) become more prominent. For example, large-amplitude oscillations were observed in the $735-765 \mathrm{~nm}$ range. These features have the characteristics of electronic coherence artifacts, and such signals persist even in pure solvent. Unfortunately, the oscillations inhibit our ability to achieve a satisfactory fit of the data near time zero, giving large $\chi^{2}$ values. Hence, where necessary, some data fits were begun at $\sim 150$ fs, i.e., after the coherence features had largely dissipated.

Although the detailed fitting and analysis of such electronic coherence features can provide information on the polarization dephasing time for a transition, ${ }^{107-110}$ for a molecule such as $\left[\mathrm{Ru}_{2} \mathrm{TIEDCl}_{4}\right]^{+}$, modeling these effects is not as simple as for a two-level system. However, since these oscillations are all observable near the time of pump and probe overlap and they dissipate quickly in the absence of the pump beam, we can speculate that the polarization dephasing time for the molecule is comparable to or slightly longer than the duration of the pump pulse ( $<200 \mathrm{fs})$. This is typical behavior for most condensedphase measurements.

\section{References and Notes}

(1) Creutz, C. Prog. Inorg. Chem. 1983, 30, 1

(2) Crutchley, R. J. Adv. Inorg. Chem. 1994, 41, 273

(3) Mountford, H. S.; Spreer, L. O.; Otvos, J. W.; Calvin, M.; Brewer, K. J.; Richter, M.; Scott, B. Inorg. Chem. 1992, 31, 5549.

(4) Mountford, H. S.; MacQueen, D. B.; Li, A.; Otvos, J. W.; Calvin, M.; Frankel, R. B.; Spreer, L. O. Inorg. Chem. 1994, 33, 1748.

(5) Spreer, L. O.; Allan, C. B.; MacQueen, D. B.; Otvos, J. W.; Calvin, M. J. Am. Chem. Soc. 1994, 116, 2187.

(6) Spreer, L. O.; Li, A.; MacQueen, D. B.; Allan, C. B.; Otvos, J. W.; Calvin, M.; Frankel, R. B.; Papaefthymiou, G. C. Inorg. Chem. 1994, $33,1753$.

(7) Zhou, J.; Li, A.; Lange, C.; Allan, C. B.; Spreer, L. O.; Otvos, J. W.; Calvin, M. Inorg. Chem. Acta 1996, 246, 241.

(8) MacQueen, D. B.; Lange, C.; Calvin, M.; Otvos, J. W.; Spreer, L. O.; Allan, C. B.; Ganse, A.; Frankel, R. B. Inorg. Chem. Acta 1997, 1997, 263.

(9) Creutz, C.; Taube, H. J. Am. Chem. Soc. 1969, 91, 3988.

(10) Robin, M. B.; Day, P. Adv. Inorg. Chem. Radiochem. 1967, 10, 247.

(11) Hush, N. S. Prog. Inorg. Chem. 1967, 8, 357

(12) Karki, L.; Williams, R. D.; Hupp, J. T.; Allan, C. B.; Spreer, L. O. Inorg. Chem. 1998, 37, 2837.

(13) Piepho, S. B.; Krausz, E. R.; Schatz, P. N. J. Am. Chem. Soc. 1978 , 100, 2996.

(14) Piepho, S. B. J. Am. Chem. Soc. 1988, 110, 6319.

(15) Piepho, S. B. J. Am. Chem. Soc. 1990, 112, 4197

(16) Root, L. J.; Ondrechen, M. J. Chem. Phys. Lett. 1982, 93, 421

(17) Ko, J.; Ondrechen, M. J. Chem. Phys. Lett. 1984, 112, 507.

(18) Ondrechen, M. J.; Ko, J.; Root, L. J. J. Phys. Chem. 1984, 88, 5919.

(19) Ko, J.; Ondrechen, M. J. J. Am. Chem. Soc. 1985, 107, 6161

(20) Ko, J.; Zhang, L.-T.; Ondrechen, M. J. J. Am. Chem. Soc. 1986, 108,1712

(21) Ondrechen, M. J.; Ko, J.; Zhang, L.-T. J. Am. Chem. Soc. 1987, 109,1672

(22) Zhang, L. T.; Ko, J.; Ondrechen, M. J. J. Am. Chem. Soc. 1987, 109,1666

(23) Petrov, V.; Hupp, J. T.; Mottley, C.; Mann, L. C. J. Am. Chem. Soc. 1994, 116, 2171.

(24) Lu, H.; Petrov, V.; Hupp, J. T. Chem. Phys. Lett. 1995, 235, 521.

(25) Krausz, E. Chem. Phys. Lett. 1985, 120, 113.

(26) Barbara, P. F.; Meyer, T. J.; Ratner, M. A. J. Phys. Chem. 1996, $100,13148$.

(27) Barker, J. R. Chem. Phys. 1983, 77, 301.

(28) Rossi, M. J.; Pladziewicz, J. R.; Barker, J. R. J. Chem. Phys. 1983 78,6695 .

(29) Barker, J. R. J. Phys. Chem. 1984, 88, 11

(30) Seilmeier, A.; Kaiser, W. Top. Appl. Phys. 1988, 60, 279.

(31) Elsaesser, T.; Kaiser, W. Annu. Rev. Phys. Chem. 1991, 42, 83

(32) Fleming, G. R.; Cho, M. Annu. Rev. Phys. Chem. 1996, 47, 109

(33) Martini, I.; Hartland, G. V. J. Phys. Chem. 1996, 100, 19764.

(34) Nesbitt, D. J.; Field, R. W. J. Phys. Chem. 1996, 100, 12735. 
(35) Schwarzer, D.; Troe, J.; Votsmeier, M.; Zerezke, M. J. Chem. Phys. 1996, $105,3121$.

(36) Eom, H. S.; Jeoung, S. C.; Kim, D.; Ha, J.-H.; Kim, Y.-R. J. Phys. Chem. A 1997, 101, 3661.

(37) Schwarzer, D.; Troe, J.; Zereske, M. J. Chem. Phys. 1997, 107 8380 .

(38) Karrlein, R.; Grabert, H. J. Chem. Phys. 1998, 108, 4972.

(39) Nakabayashi, T.; Okamoto, H.; Tasumi, M. J. Phys. Chem. A 1998, 102,9686

(40) Schwarzer, D.; Troe, J.; Zerezke, M. J. Phys. Chem. A 1998, 102, 4207.

(41) Gottfried, N. H.; Seilmeier, A.; Kaiser, W. Chem. Phys. Lett. 1984 $111,326$.

(42) Seilmeir, A.; Scherer, P. O. J.; Kaiser, W. Chem. Phys. Lett. 1984, 105,140 .

(43) Wondrazek, F.; Seilmeir, A.; Kaiser, W. Chem. Phys. Lett. 1984, 104, 121.

(44) Wild, W.; Seilmeir, A.; Gottfried, N. H.; Kaiser, W. Chem. Phys. Lett. 1985, 119, 259.

(45) Hutchinson, J. S.; Marshall, K. T. J. Chem. Soc., Faraday Trans. $2 \mathbf{1 9 8 8}, 84,1535$.

(46) Marshall, K. T.; Hutchinson, J. S. J. Chem. Phys. 1991, 95, 3232. 321 .

(47) Rosker, M. J.; Wise, F. W.; Tang, C. L. Phys. Rev. Lett. 1986, 57,

(48) Chesnoy, J.; Mokhtari, A. Phys. Rev. A 1988, 38, 3566.

(49) Walmsley, I. A.; Mitsunaga, M.; Tang, C. L. Phys. Rev. A 1988 38,4681 .

(50) Fain, B.; Lin, S. H. J. Chem. Phys. 1990, 93, 6387.

(51) Pollard, W. T.; Dexheimer, S. L.; Wang, Q.; Peteanu, L. A.; Shank,

C. V.; Mathies, R. A. J. Phys. Chem. 1992, 96, 6147. 497.

(52) Pollard, W. T.; Mathies, R. A. Annu. Rev. Phys. Chem. 1992, 43,

(53) Vos, M. H.; Rappaport, F.; Lambry, J.-C.; Breton, J.; Martin, J.-L. Nature 1993, 363, 320.

(54) Banin, U.; Kosloff, R.; Ruhman, S. Chem. Phys. 1994, 183, 289.

(55) Chachisvilis, M.; Pullerits, T.; Jones, M. R.; Hunter, C. N.;

Sundstrom, V. Chem. Phys. Lett. 1994, 224, 345.

(56) Dhar, L.; Rogers, J. A.; Nelson, K. A. Chem. Rev. 1994, 94, 157.

(57) Wang, Q.; Schoenlein, R. W.; Peteanu, L. A.; Mathies, R. A.; Shank,

C. V. Science 1994, 266, 422.

(58) Reid, P. J.; Silva, C.; Barbara, P. F.; Karki, L.; Hupp, J. T. J. Phys. Chem. 1995, 99, 2609.

(59) Wynne, K.; Reid, G. D.; Hochstrasser, R. M. J. Chem. Phys. 1996, $105,2287$.

(60) Hayashi, M.; Yang, T.-S.; Mebel, A.; Chang, C. H.; Lin, S. H.; Scherer, N. F. Chem. Phys. 1997, 217, 259.

(61) Zhu, L.; Widom, A.; Champion, P. M. J. Chem. Phys. 1997, 107, 2858.

(62) Hayashi, M.; Yang, T.-S.; Yu, J.; Mebel, A.; Chang, R.; Lin, S.

H.; Rubtsov, I. V.; Yoshihara, K. J. Phys. Chem. A 1998, 102, 4256.

(63) Wolfseder, B.; Seider, L.; Domcke, W.; Stock, G.; Seel, M.;

Engleitner, S.; Zinth, W. Chem. Phys. 1998, 233, 323

(64) Rubtsov, I. V.; Yoshihara, K. J. Phys. Chem. A 1999, 103, 10202.

(65) Shapiro, M.; Vrakking, M. J. J.; Stolow, A. J. Chem. Phys. 1999

110,2465

(66) Yang, T.-S.; Chang, M.-S.; Chang, R.; Hayashi, M.; Lin, S. H.;

Vohringer, P.; Dietz, W.; Scherer, N. F. J. Chem. Phys. 1999, 110, 12070.

(67) Jonas, D. M.; Bradforth, S. E.; Passino, S. A.; Fleming, G. R. J.

Phys. Chem. 1995, 99, 2594.

(68) Johnson, A. E.; Myers, A. B. J. Chem. Phys. 1996, 104, 2497.

(69) Diffey, W. M.; Homoelle, B. J.; Edington, M. D.; Beck, W. F. J.

Phys. Chem. B 1998, 102, 2776.

(70) Bixon, M.; Jortner, J. J. Chem. Phys. 1997, 107, 1470.

(71) Mebel, A. M.; Hayashi, M.; Lin, S. H. Chem. Phys. Lett. 1997 274, 281.

(72) Peluso, A.; Santoro, F.; Del Re, G. Int. J. Ouantum Chem. 1997, 63,233

(73) Hayashi, M.; Mebel, A. M.; Liang, K. K.; Lin, S. H. J. Chem. Phys. 1998, 108, 2044.

(74) Wynne, K.; Hochstrasser, R. M. Coherence and Adiabaticity in Electron Transfer. In Electron Transfer-from Isolated Molecules to Biomolecules Part 2; Jortner J., Bixon, M., Eds.; John Wiley and Sons: New York, 1999; p 263.

(75) Huang, Y.; Rettner, C. T.; Auerbach, D. J.; Wodtke, A. M. Science 2000, 290, 111.
(76) Trushin, S. A.; Fuss, W.; Schmid, W. E.; Kompa, K. L. J. Phys. Chem. A 1998, 102, 4129.

(77) Joo, T.; Jia, Y.; Yu, J.-Y.; Jonas, D. M.; Fleming, G. R. J. Phys. Chem. 1996, 100, 2399.

(78) Kumble, R.; Palese, S.; Visschers, R. W.; Dutton, P. L.; Hochstrasser, R. M. Chem. Phys. Lett. 1996, 261, 396.

(79) Lin, S. H. J. Chem. Phys. 1966, 44, 3759.

(80) Lin, S. H.; Bersohn, R. J. Chem. Phys. 1968, 48, 2732.

(81) Freed, K. F.; Jortner, J. J. Chem. Phys. 1970, 52, 1272.

(82) Fischer, S. F. Chem. Phys. Lett. 1971, 11, 577.

(83) Nitzan, A.; Jortner, J. J. Chem. Phys. 1971, 55, 1355.

(84) Siebrand, W. J. Chem. Phys. 1971, 54, 363.

(85) Heller, F. F.; Freed, K. F.; Gelbart, W. M. J. Chem. Phys. 1972 $56,2309$.

(86) Fischer, G. Vibronic Coupling-The Interaction between the Electronic and Nuclear Motions; Academic Press: London, 1984.

(87) Wynne, K.; Galli, C.; Hochstrasser, R. M. J. Chem. Phys. 1994, $100,4797$.

(88) Herzberg, G. Electronic Spectra and Electronic Structure of Polyatomic Molecules; D. Van Nostrand Reinhold: New York, 1966; Vol. 3 .

(89) Shriver, D. F.; Dunn, J. B. R. Appl. Spectrosc. 1974, 28, 319.

(90) Heller, E. J. Acc. Chem. Res. 1981, 14, 368.

(91) Heller, E. J.; Sundberg, R. L.; Tannor, D. J. Phys. Chem. 1982 86,1822 .

(92) Tannor, D. J.; Heller, E. J. J. Chem. Phys. 1982, 77, 202.

(93) Myers, A. B.; Mathies, R. A. Resonance Raman Intensities: A Probe of Excited-State Structure and Dynamics. In Biological Applications of Raman Spectroscopy; Spiro, T. B., Ed.; John Wiley and Sons: New York, 1987; Vol. 2; p 1.

(94) Shin, K.-S. K.; Zink, J. I. Inorg. Chem. 1989, 28, 4358.

(95) Zink, J. I.; Shin, K.-S. K. Molecular Distortions in Excited Electronic States Determined from Electronic and Resonance Raman Spectroscopy. In Advances in Photochemistry; Volman, D. H., Hammond, G. S., Neckers, D. C., Eds.; John Wiley and Sons: New York, 1991; Vol. 16, p 119.

(96) Myers, A. B. Excited Electronic State Properties from GroundState Resonance Raman Intensities. In Laser Techniques in Chemistry; Myers, A. B., Rizzo, T. R., Eds.; John Wiley and Sons: New York, 1995; Vol. XXIII, p 325.

(97) Myers, A. B. Chem. Rev. 1996, 96, 911

(98) Myers Kelly, A. B. J. Phys. Chem. A 1999, 103, 6891.

(99) Frisch, M. J.; Trucks, G. W.; Schlegel, H. B.; Scuseria, G. E.; Robb, M. A.; Cheeseman, J. R.; Zakrzewski, V. G.; Montgomery, J. A., Jr.; Stratmann, R. E.; Burant, J. C.; Dapprich, S. J.; Millam, M.; Daniels, A. D.; Kudin, K. N.; Strain, M. C.; Farkas, O.; Tomasi, J.; Barone, V.; Cossi, M.; Cammi, R.; Mennucci, B.; Pomelli, C.; Adamo, C.; Clifford, S.; Ochterski, J.; Petersson, G. A.; Ayala, P. Y.; Cui, Q.; Morokuma, K.; Malick, D. K.; Rabuck, A. D.; Raghavachari, K.; Foresman, J. B.; Cioslowski, J.; Ortiz, J. V.; Baboul, A. G.; Stefanov, B. B.; Liu, G.; Liashenko, A.; Piskorz P.; Komaromi, I.; Gomperts, R.; Martin, R. L.; Fox, D. J.; Keith, T.; AlLaham, M. A.; Peng, C. Y.; Nanayakkara, A.; Gonzalez, C.; Challacombe, M.; Gill, P. M. W.; Johnson, B.; Chen, W.; Wong, M. W.; Andres, J. L.; Gonzalez, C.; Head-Gordon, M.; Replogle, E. S.; Pople, J. A. Gaussian 98W, Revision A.7, 5.2 ed.; Gaussian, Inc.: Pittsburgh, PA, 1998.

(100) Mukamel, S.; Yan, Y. J. In Recent Trends in Raman Spectroscopy; Banerjee, S. B. J., S. S., Ed.; World Scientific: Singapore, 1989; p 160 (101) Bosma, W. B.; Yan, Y. J.; Mukamel, S. Phys. Rev. A 1990, 42, 6920.

(102) Mukamel, S. Annu. Rev. Phys. Chem. 1990, 41, 647.

(103) Spears, K. G. J. Phys. Chem. 1995, 99, 2469.

(104) Huang, C. P.; Kapteyn, H. C.; McIntosh, J. W.; Murnane, M. M. Opt. Lett. 1992, 17, 139.

(105) Squier, J.; Korn, G.; Mourou, G. Opt. Lett. 1993, 18, 625.

(106) Skhremitchev, B.; Wang, C.; Walker, G. C. Rev. Sci. Instrum. 1996, 67, 3799 .

(107) Cruz, C. H. B.; Gordon, J. P.; Becker, P. C.; Fork, R. L.; Shank, C. V. IEEE J. Quantum Electron. 1988, 24, 261.

(108) Zinth, W.; Kaiser, W. Top. Appl. Phys. 1988, 60, 279 67 .

(110) Lucke, A.; Mak, C. H.; Egger, R.; Ankerhold, J.; Stockburger, J.; Grabert, H. J. Chem. Phys. 1997, 107, 8397. 